Research, part of a Special Feature on Empirical based agent-based modeling

\title{
Companion Modeling, Conflict Resolution, and Institution Building: Sharing Irrigation Water in the Lingmuteychu Watershed, Bhutan
}

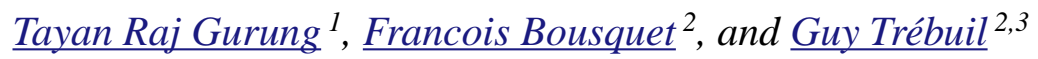

\begin{abstract}
We used multi-agent systems (MAS), following the companion modeling method, to facilitate water management negotiations in Bhutan. We show how this methodology helped resolve a conflict over the sharing of water resources by establishing a concrete agreement and creating an institution for collective watershed management. The conceptual model begins with a role-playing game (RPG). The stakeholders play the game, thus validating the proposed environment, the behavioral rules, and the emergent properties of the game. It is then relatively easy to translate the RPG into computerized MAS that allow different scenarios to be explored. After this first step in the MAS model, stakeholders then create an institution. A second model is developed to facilitate this process. We conclude by discussing the relationship between the models and reality, as well as the use of MAS as a mediation tool and the social process.
\end{abstract}

Key Words: Bhutan, companion modeling; multi-agent system; role-playing game; watershedmanagement

\section{INTRODUCTION}

Several approaches for supporting the collective management of ecosystems, such as adaptive management (Holling 1978, Walters and Hilborn 1978) and co-management (Berkes 1997, BorriniFeyerabend et al., 2000), have been developed in the recent past. These approaches recognize that management does not only consist of understanding the state of the ecosystem and its dynamics, but it also deals with the social process leading to this ecological state and the social processes that may lead to other states. In other words, what is important are the solutions emerging from interactions among the different stakeholders. And with them comes a different portfolio of interventions, including mediation to resolve conflicts, facilitation of learning, and participatory approaches that involve people in negotiating collective action. The relationships between simulation models and collective decision making in natural resource management occupy a large part of the literature on adaptive management (Holling 1978, Walters and Hilborn 1978). However, the model is often a biophysical one, and few papers (Abel 1999, Costanza and Ruth 1998, Lynam et al., 2002) mention the participation of stakeholders from different organizational levels in the modeling steps (from conceptualization to scenario simulation). Participatory geographic information systems (GIS) have demonstrated the ability of many illiterate people to use high-tech tools (Gonzalez 2000). This paper presents the use of a multi-agent systems (MAS) model to facilitate negotiation between conflicting stakeholders in a Bhutanese watershed, leading to the creation of a formal institution. This model was conceptualized and discussed with the stakeholders according to a methodology called "companion modeling." The different steps of the process are described, from the presentation of the context through to the creation of a watershed committee, and a discussion follows on the use of empirical models within this conflictresolution and institution-building process. The relationship between the model and the reality, the use of the model, and the social process are discussed.

In brief, the main principle of the companion modeling (ComMod) approach is to develop simulation models integrating various stakeholders' points of view and to use them within the context

\footnotetext{
${ }^{1}$ RNR Research Centre, Council for RNR Research of Bhutan, Ministry of Agriculture, Bhutan, ${ }^{2}$ CIRAD Green TA 60/15 Campus de Baillarguet 34398 Montpellier Cedex 5 France, ${ }^{3}$ CU-Cirad Project Department of Biology Faculty of Science Chulalongkorn University Phayathai Road, Pathumwan Bangkok 10330 Thailand
} 
of the stakeholders' platform (Röling 1996) for collective learning. This is a modeling approach in which stakeholders participate fully in the construction of models to improve their relevance and increase their use for the collective assessment of scenarios. The general objective of ComMod is to facilitate dialogue, shared learning, and collective decision making through interdisciplinary and "implicated" research to strengthen the adaptive management capacity of local communities. By using such an approach, we expect to be in a better position to deal with the increased complexity of integrated natural resource management (INRM) problems, their evolving and continuous characteristics, and the increased rapidity of evolutions and changes in number of stakeholders.

We use MAS tools in a cyclic ComMod process displayed in Fig. 1. It is made up of three stages that can be repeated as many times as needed:

1. Field investigations and a literature search on the observed world supply information and help generate explicit hypotheses for modeling.

2. Modeling, i.e., the conversion of existing knowledge into a formal tool to be used as a simulator.

3. Simulations, conducted according to an experimental protocol either on a computer or through a role-playing game (RPG), to challenge the former understanding of the system and to identify new key questions for new focused investigations in the field.

Thus, the MAS model can be implemented either through a computerized model or through a RPG. We named this process "companion modeling" because it is used in the mediation process (the social dimension of the companion) and it co-evolves with this social process (temporal and adaptive dimensions). The next question concerned how to use these models in an interactive way with stakeholders. In agreement with the abovementioned principles, a model, which is a given kind of representation among other possible ones, should be presented in an explicit and transparent way to avoid, as much as possible, the "black box effect" when it is proposed to users. We were inspired by the work of several scientists working in the field of environmental management who developed and used RPGs for collective learning or collective action (Burton 1994, Meadows and Meadows 1993, Mermet 1993, Piveteau 1994). Intuitively, a MAS model could be seen as an RPG simulated by the computer. Consequently, we proposed setting up RPGs, similar to MAS models (Bousquet et al., 1999, Bousquet et al., 1996), with the objective of inviting real stakeholders to play the game in order for them to:

- understand the model, and more precisely, understand the difference between the model and reality;

- validate it by examining the individual behaviors of agents, their interactions, and the properties of the system emerging from their interactions, and by proposing modifications to behavior or interactions,

- be able to follow MAS simulations on the computer, and propose scenarios to be assessed and discussed following their simulations.

Several applications have been already developed (d'Aquino et al. 2003, Barnaud et al. 2006, Barreteau and Bousquet 2000, Castella et al. 2005, Étienne 2003, Étienne et al. 2003, Lynam et al. 2002).

We propose distinguishing between two specific contexts when using this approach: the production of knowledge on a given complex system and the support to collective decision-making processes. Although the first context deals with systems research via a particular relationship to field work, the second one corresponds to methodological research to facilitate the concerted management of such systems. In the first case, the key ComMod challenge is to deliver an improved understanding of the interacting processes related to the resource management problem being examined rather than a "roadmap" itinerary for renewable resource management. In the latter case, even if it is not covering the whole process of mediation by itself, ComMod is contributing significantly to it. This approach intervenes upstream of any technical decision to support the deliberation of concerned actors, to produce a shared representation of the problem at stake, and to identify possible ways toward collective management and alleviation of the problem. Meanwhile, ComMod does not include the other possible steps of the mediation process, 
Fig. 1. The companion modeling process.

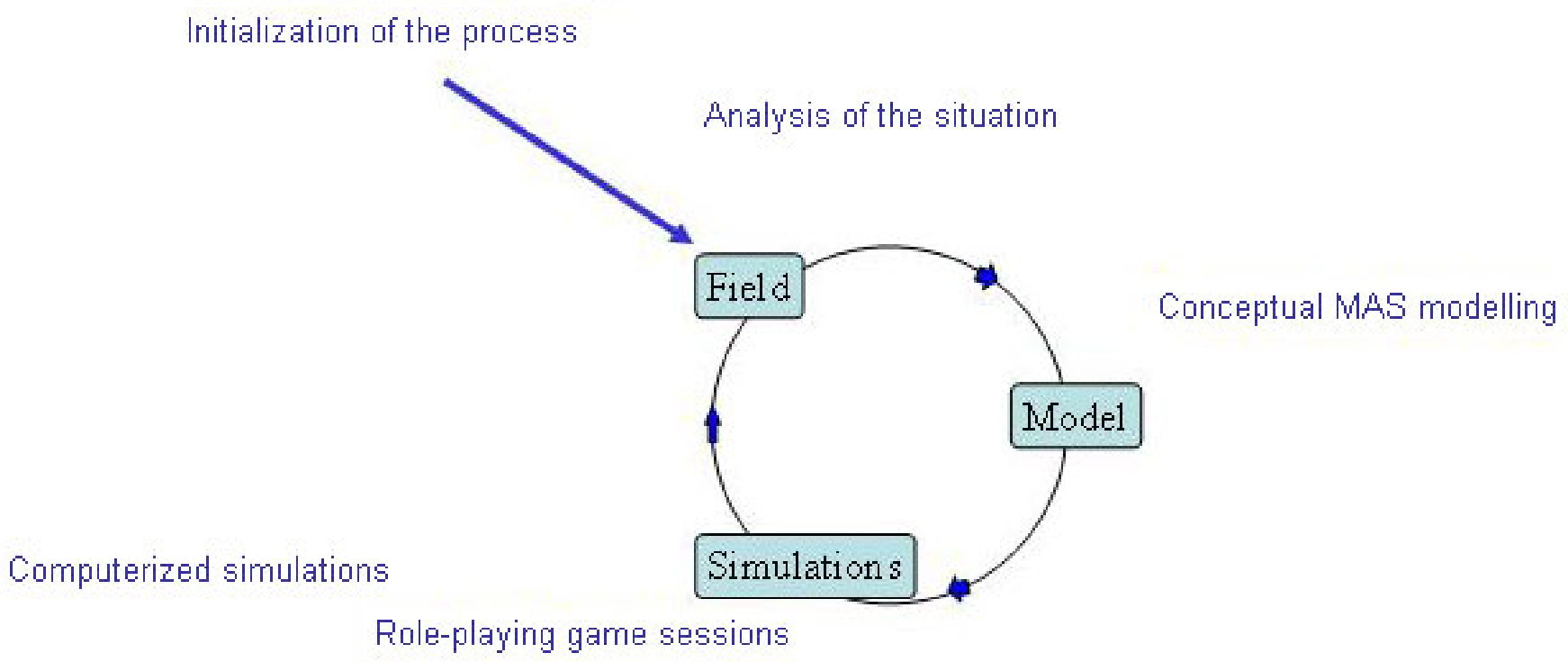

particularly those dealing with more quantified expertise (type and size of a new infrastructure, estimation of production and costs, etc.).

\section{THE PROBLEM: CONFLICT OVER WATER MANAGEMENT}

\section{General Context of Natural Resource Management in Bhutan}

Bhutan is predominantly an agrarian nation, with some $80 \%$ of the population dependent on smallscale mountain agriculture and livestock rearing for their livelihood. Bhutan has successfully maintained its $72 \%$ forest cover, rich biodiversity, and plentiful water resources (Royal Government of Bhutan 2003). In Bhutan, age-old traditions and wellestablished relationships among users constituted a broadly respected customary regime of natural resource management, which has resulted from the blending of appreciation for the dependence of people on natural resources and the value of these resources (National Environment Commission 1998). However, over the years, the role and efficiency of these local norms and arrangements have weakened because of the influence of development and commercialization.

The ratification of the Forest Act of 1969 showed that Bhutan was already concerned about INRM problems. However, Gurung and Turkelboom (2000), Messerschmidt et al. (2001), and Tshering (2001) suggest that, since the centralization of forest resource management in 1969, many of the indigenous knowledge systems and communitybased regimes for natural resource management have disappeared, as communities lost their customary rights and control over local forest resources. This has brought about an "open access" regime, as adequate resources were not in place to effectively and efficiently implement the forest regulations (Ministry of Agriculture 2002a). Many natural resources are considered to be under the purview of the Forest Act. However, the specificity of the rules varies among the resources. For instance, there is no specific policy and law for water resources; the Ministry of Agriculture (MoA) is currently drafting the Water Act. This act will address the policy, legal, and organizational framework for the fair sharing of resources, for property rights (including water rights), and for 
effective participation, partnerships, and cooperation of stakeholders, as well as conflict avoidance (Bhutan Water Partnership 2003).

According to the decentralization policy, beneficiary participation is the primary driving force for development. Furthermore, with the ratification of Dzongkhag Yargey Tshogtshung (DYT) (District Development Committee) and Geog Yargey Tshogtshung (GYT) (Block Development Committee) governance acts, the responsibility for managing natural resources has been passed on to communities and local institutions. This is specifically a devolution of decision making to the lowest appropriate level (Röling 1999). To complement the devolution of INRM responsibilities, the MoA formulated and released a community-based natural resource management (CBNRM) framework in 2002 (MoA 2002a).

As changes in resource use are supposed to emerge from human learning, interactions, and institutions, these changes often require considerable attention to create a common perspective on problems, diagnosis, and possible solutions. Therefore, an integrated approach is needed to understand resource-use dynamics as this often involves multiple stakeholders and a series of decisions emerging from different behavioral patterns.

One of the natural resources that has been principally managed by traditional institutions and norms is water (Litmus Consult 2002). Access to water and management are still governed by traditional rules that evolved during times when water demand was limited (MoA 2002a). A nationwide renewable natural resources census indicated that $21 \%$ of 60000 farmers interviewed reported a lack of irrigation water as a major constraint to agricultural production, second only to crop predation by wild animals (42\%) (MoA $2002 \mathrm{~b}$ ). With increasing demand and competition for water, frequent violent confrontations and abuse of resources have become a major concern (Renewable Natural Resources Research Center 1998).

\section{Case Study: Lingmuteychu Watershed}

The Renewable Natural Resources Research Center (RNRRC) in Bajo conducted preliminary diagnostic studies in the Lingmuteychu watershed in 1997 as part of the community-based natural resource management research (Renewable Natural Resources Research Center 1998). This study identified numerous constraints to low crop production in the watershed, of which lack of irrigation water during transplanting was reported as a major problem. Considering the problems and existing field experiences, the site was selected for this research. Lingmuteychu is a small watershed located at $27^{\circ}$ $33^{\prime} \mathrm{N}$ and $89^{\circ} 55^{\prime} \mathrm{E}$ on the east bank of the Punatshang Chu river in west-central Bhutan, occupying an area of $34 \mathrm{~km}^{2}$ (Fig. 2). It is drained by the $11 \mathrm{~km}$ long Limti Chu stream that originates as a spring from a rock face at an altitude of 2400 $\mathrm{m}$ north of Limbukha village. It is a rain-fed stream because the ranges that confine the watershed are below the snow line. The stream serves five irrigation systems, supporting 11 irrigation channels that irrigate about 180 ha of terraced wetland belonging to 162 households of six villages (Renewable Natural Resources Research Center 1998). These six villages share irrigation water within a broadly respected customary regime. The two villages of Limbukha and Dompola, situated approximately $3 \mathrm{~km}$ apart upstream of the Lingmuteychu watershed, are in perennial conflict over sharing irrigation water.

There are five major irrigation networks in the Lingmuteychu watershed. They are Limbukha, Dompola, Omteykha, Matalumchu, and Wangjokha/ Bajothangu. The first four schemes derive water from the Limtichu stream; Wangjokha/Bajothangu is irrigated by Bajo canal, which brings water from another watershed. As four major channels depend on one source of water, this increases the conflict over access to the water. In principle, based on traditional rules, the upstream communities have greater control over water and tend to hold water for a longer time. In such a situation, the downstream communities have to satisfy their needs with their agreed-upon share. However, there are cases of water theft too. As most of the canals are earthen without concrete lining, the conveyance efficiency of these canals is reported to be only $40 \%$, which is extremely low.

The base flow during the dry months of April and May fluctuates from about 40 to $50 \mathrm{~L}^{-1}$. The flow produced by a widespread rain in the watershed can be more than $500 \mathrm{~L}^{-1}$. The rainfall-runoff response is quick, and the stream returns to its base flow within a couple of days after the rainfall. The fluctuating nature of the stream mainly results from 
Fig. 2. Map of the study area: Lingmuteychu watershed, Punakha District, West-central Bhutan.

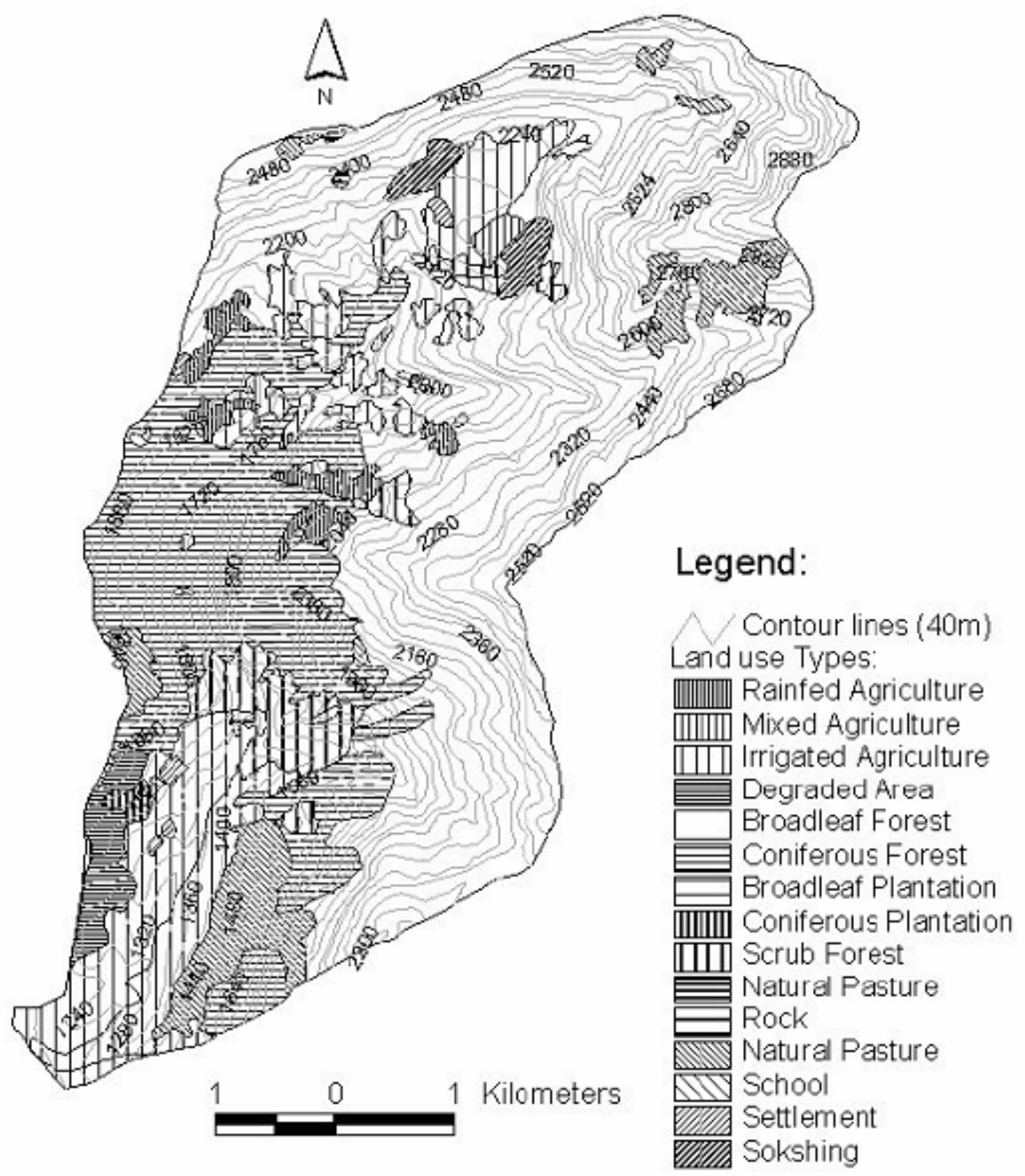


the steep gradient of the watershed. The watershed receives an average annual rainfall of $700 \mathrm{~mm}$ (Renewable Natural Resources Research Center 1998). Regulations in terms of water diversion by different irrigation canals from the Limti Chu are based on two broad principles. The rule "first come, first served" applies, which means that existing schemes have an established water right and can prevent newcomers from using it. For instance, Nabche (one of the villages within the watershed) is a resettled community and does not have access to water, which prevents villagers from constructing an irrigation canal. The second rule can be interpreted as "more water for upstream communities." Conflicts arise mainly from these two rules. Under such a water-use regime, the community in the uppermost catchment (Limbukha), close to the intake point, has absolute control over the headwater.

Ironically, Dompola, the second village in the upper catchment, located approximately $3 \mathrm{~km}$ downstream from the intake point, does not have direct access to the stream. Dompola has to share water with Limbukha, and the water release date and volume of water diverted from the stream are strictly adhered to. According to the traditional arrangement, Dompola gets half of the stream flow only from the $10^{\text {th }}$ day of the $5^{\text {th }}$ Bhutanese month every year. However, even after this date, Limbukha farmers still use water from Dompola's share to irrigate their land. Therefore, Dompola farmers struggle to get their paddy field transplanted. This indiscriminate use of water in the upper catchment results in conflict between the two villages.

Within a village, water is shared on the basis of a rotation system locally known as "chukor." The rotation interval among different communities in the watershed varies from 3 to 13 days. In Limbukha and Dompola, water is shared on the basis of four categories: "Thruelpa," "Cheep," "Chatro," and "Lhangchu." These categories correspond to the following division of access to irrigation water:

- a thruelpa is entitled to half the flow in the canal ( $1 / 2$ of the canal flow),

- a cheep is entitled to half of a thruelpa ( $1 / 4$ of the canal flow),

- a chatro is entitled to half of a cheep $(1 / 8$ of the canal flow),
- a lhangchu has no entitlement and must beg for water.

The existing water rights are not equitable. As the water resource becomes scarce, the current system has deficiencies. With differences in water rights, conflict can emerge within and between communities. It has also been shown that farmers use excessive amount of water (MoA 2002a). This is aggravated by the introduction of multiplecropping practices in upper villages, which have significant effects on the water supply and rice production in the lower community (Renewable Natural Resources Research Center 1997).

\section{THE COMPANION MODELING PROCESS}

\section{Preliminary Analysis}

Primary and secondary data were collected. Secondary data were extracted from various published and unpublished reports, journals, literature reviews, proceedings, personal communications, key informants, and personal observations. Analysis of secondary data helped focus this research. Institutions such as the Research Center in Bajo, the District and Block Agriculture Office, and the Planning and Policy Division (PPD) of the MoA provided both formal and informal information. The objective was to identify the issues at stake, the dynamics of the resource, and the behavior of the stakeholders. Primary data were collected using formal and informal methods. The basic purpose of the primary data collection was to make a systematic diagnosis of the watershed and farming system aspects related to the problem under study, and to subsequently help in designing the RPG. Initially, informal visits were made to the site and discussions were held with the administrators, researchers, extension staff, community leaders, and some farmers. These discussions bettered our understanding of the problem and helped us conceptualize the study. A formal household survey was conducted using a structured questionnaire that was developed based on a preliminary analysis of the secondary data and the basic information needed for designing a RPG. The questionnaire was pretested in Limbukha, and was then followed by a survey of 40 households from the two villages. The household survey was designed to collect data in three major areas: general socioeconomic information, social organization, and irrigation water management. 
Considering that the farmers in the watershed operate in a diverse socioeconomic and resourceconstrained situation, although geographically small in extent, it is critical to understand their farming objectives, the farm environment in which they operate, their management choices, and possible improvements. As suggested by Trébuil et al. (1997), in order to study the functioning of farming systems, five aspects need to be analyzed: (i) the family situation, the size of the farming system and its objectives; (ii) the farm environment; (iii) the strategy for earning a livelihood; (iv) the mix of farm activities, and their technical and economic performance; and (v) the potential for improvement. Four farm types, corresponding to the FAO's farm classification (McConnell and Dillon. 1997), were identified in Lingmuteychu watershed as: small independent specialized commercial farms (Type 1); small independent specialized partcommercial family farms (Type 2); small semisubsistence or part-commercial family farms (Type 3 ); and small subsistence-oriented family farms (Type 4). These four objectives match almost precisely with the four water-sharing categories of villagers in the watershed.

Depending on their categories, each type of farm has a unique choice of production and economic activities, and subsequently of management options. The environment in which they function is, to a large extent, similar and is characterized by a shortage in water supply and labor, damage by wild animals, and limited access to markets. From the analysis of differences in the functioning of farming systems, key parameters were identified to distinguish fairly precisely the differences between the four types and subtypes. Major production choices, related management options, and access to irrigation water were used to classify farm types. The classification of four farm types was used to further group farms of two villages (Table 1). According to the farm typology, $37 \%$ of the farms can be categorized as Type 1 ; similarly, $26 \%$ as Type $2 ; 28 \%$ as Type 3 , and $8 \%$ as Type 4 . The analysis also showed that a higher percentage of farms control a larger share of irrigation water, particularly in Limbukha. This could lead to disparity in access to irrigation water. Given that irrigation is an important input for irrigated rice, accessing irrigation water at the right time and at the right volume is of paramount importance. Farm Type 1, with full access to water at the appropriate time, has the advantage. In contrast, $30 \%$ of the farms in Dompola have to share half of the irrigation flow, which increases the conflict for water. The Type 4 farm, which represents $8 \%$ of the farms, has to depend on other farmers for water. Basically, they have to exchange water for labor, which further puts the Type 4 farmer under pressure to get water.

\section{Collective Workshop to Facilitate Discussion between Two Villages ${ }^{[1]}$}

\section{Description of role-playing game}

Considering the problem of conflict over irrigation water sharing between two villages (Limbukha, an upstream village, and Dompola, a downstream village), the RPG method was conceived as a potential tool to initiate and facilitate dialogue between the two villages and, for the researchextension team, to enhance its understanding of the problem. The RPG was developed by the researchextension team based at the RNRRC. The objective is to provide a virtual environment in which the farmers may make decisions, similar to reality but simple enough to be played (Fig. 3). It took about 3 weeks to be developed and was pre-tested by the researchers at the research station. With the onset of the transplanting season from the fourth Bhutanese month (end of May), Limbukha farmers started transplanting in the watershed. The game started on the $10^{\text {th }}$ day of the $5^{\text {th }}$ month, coinciding with when the Limbukha farmers release $50 \%$ of the water into the Dompola canal. Six farmers each from two villages were categorized according to their water-right categories for the game. Six farmers of Limbukha village had yet to transplant rice, which meant that what they did would still have an effect (on the quantity of water available for the next village, hence on the actions of the farmers in the next village). There were two major chance factors: rainfall (normal and low) and market price (high and low). Rainfall was declared after drawing a card at the start of the game, whereas the market price was declared after each game. Each crop year was divided into two cycles (first week of June to October and third week of June to October). Therefore, each successive time step in a given season covered roughly two water-share cycles (12 days each) from the $10^{\text {th }}$ day of the $5^{\text {th }}$ month to the $4^{\text {th }}$ day of the $6^{\text {th }}$ month $(=$ end of the rice transplanting season). The complete description of the RPG is in Append. 1. 
Table 1. Share of irrigation water used by different farm types in two villages of the Lingmuteychu watershed

\begin{tabular}{|c|c|c|c|c|c|c|c|}
\hline \multirow[t]{2}{*}{ Typology } & \multirow{2}{*}{$\begin{array}{l}\text { Land hold- } \\
\text { ing (ha) }\end{array}$} & \multirow{2}{*}{ Water share } & \multirow[t]{2}{*}{ Income source } & \multirow[t]{2}{*}{ Management choice } & \multicolumn{2}{|c|}{$\%$ Farms $(n=49)$} & \multirow{2}{*}{$\begin{array}{l}\text { Farmer ca- } \\
\text { tegory }\end{array}$} \\
\hline & & & & & $\begin{array}{l}\text { Limbukha } \\
(n=33)\end{array}$ & $\begin{array}{c}\text { Dompola } \\
(n=16)\end{array}$ & \\
\hline $\begin{array}{l}\text { Type I: Small } \\
\text { independent specialized } \\
\text { commercial farms }\end{array}$ & $1.2-1.5$ & $\begin{array}{l}\text { Full flow of } \\
\text { canal }\end{array}$ & $\begin{array}{l}\text { Potato and } \\
\text { vegetables }\end{array}$ & $\begin{array}{l}\text { Mechanization, } \\
\text { fertilizer, and } \\
\text { pesticides }\end{array}$ & 35 & 2 & Thruelpa \\
\hline $\begin{array}{l}\text { Type II: Small } \\
\text { independent specialized } \\
\text { part-commercial family } \\
\text { farm }\end{array}$ & $0.3-2.4$ & $\begin{array}{l}\text { Half of } \\
\text { Thruelpa's } \\
\text { share }\end{array}$ & $\begin{array}{l}\text { Potato and } \\
\text { vegetable }\end{array}$ & $\begin{array}{l}\text { Manual and animal } \\
\text { power, chemical } \\
\text { fertilizer }\end{array}$ & 16 & 10 & Cheep \\
\hline $\begin{array}{l}\text { Type III: Small semi- } \\
\text { subsistence or part- } \\
\text { commercial family } \\
\text { farms }\end{array}$ & $0.15-1.4$ & $\begin{array}{c}\text { Half of } \\
\text { Cheep's share }\end{array}$ & $\begin{array}{l}\text { Potato, veget- } \\
\text { ables, off- } \\
\text { farm, dairy }\end{array}$ & $\begin{array}{l}\text { Manual and animal } \\
\text { power }\end{array}$ & 8 & 20 & Chatro \\
\hline $\begin{array}{l}\text { Type IV: Small } \\
\text { subsistence oriented } \\
\text { farm }^{\mathrm{a}}\end{array}$ & $0-0.6$ & No share & Off farm & $\begin{array}{l}\text { Share cropping, } \\
\text { manual }\end{array}$ & 8 & 0 & Lhangchu \\
\hline
\end{tabular}

\section{Playing the game}

Two sessions of role-playing games were organized in May and December 2003 in Dompola. The second session of the game, played in December, was basically the same game with provision for sharing water against labor and involvement of development committee members as observers of the game session.

In Dompola, the RPG was used for 3 days. Six farmers from each of the two villages representing four water-share categories (Thruelpa, Cheep, Chatho, and Lhangchu) were selected to play the game. Players were given predefined numbers of rice fields (each field size was 1 langdo $(=0.1 \mathrm{ha})$ : Thruelpa had eight plots, Cheep had six, Chatho had four, and Lhangchu only two.

The first day was assigned for RPG sessions, which started with a briefing about the game, the purpose, the role of the players, and the expected outputs. The game sessions corresponded to three different modes of communication among villages: intravillage, intervillage (collective), and swapping roles. The first session represented the existing situation, in which each village discussed water sharing independently at the village level and accordingly decided to grow different crops. Even the game boards were kept in distant places such that one village could not see the actions of the other village. The game was played for seven cropping seasons. During the second session, farmers from both villages formed one group to discuss collectively sharing water between the two villages. The game boards were placed together side by side to allow the farmers to see and discuss them. This was necessary to demonstrate that two villages can freely discuss and share water to grow crops for five crop cycles in a collective decision mode. During the third session, roles were swapped between the two villages. It was anticipated that this would provide a better understanding of the other village's situation, identify any unique decisions, and bring about new understanding as a result of the role swapping.

The second day was devoted to analyzing the RPG outputs and discussing them among the facilitators. On the third day, based on the preliminary analysis 
Fig. 3. The game board for one village: one column for each farmer (farmer A has 8 plots, farmer B has two plots). Yellow papers represent potato crops and pink papers represent rice.

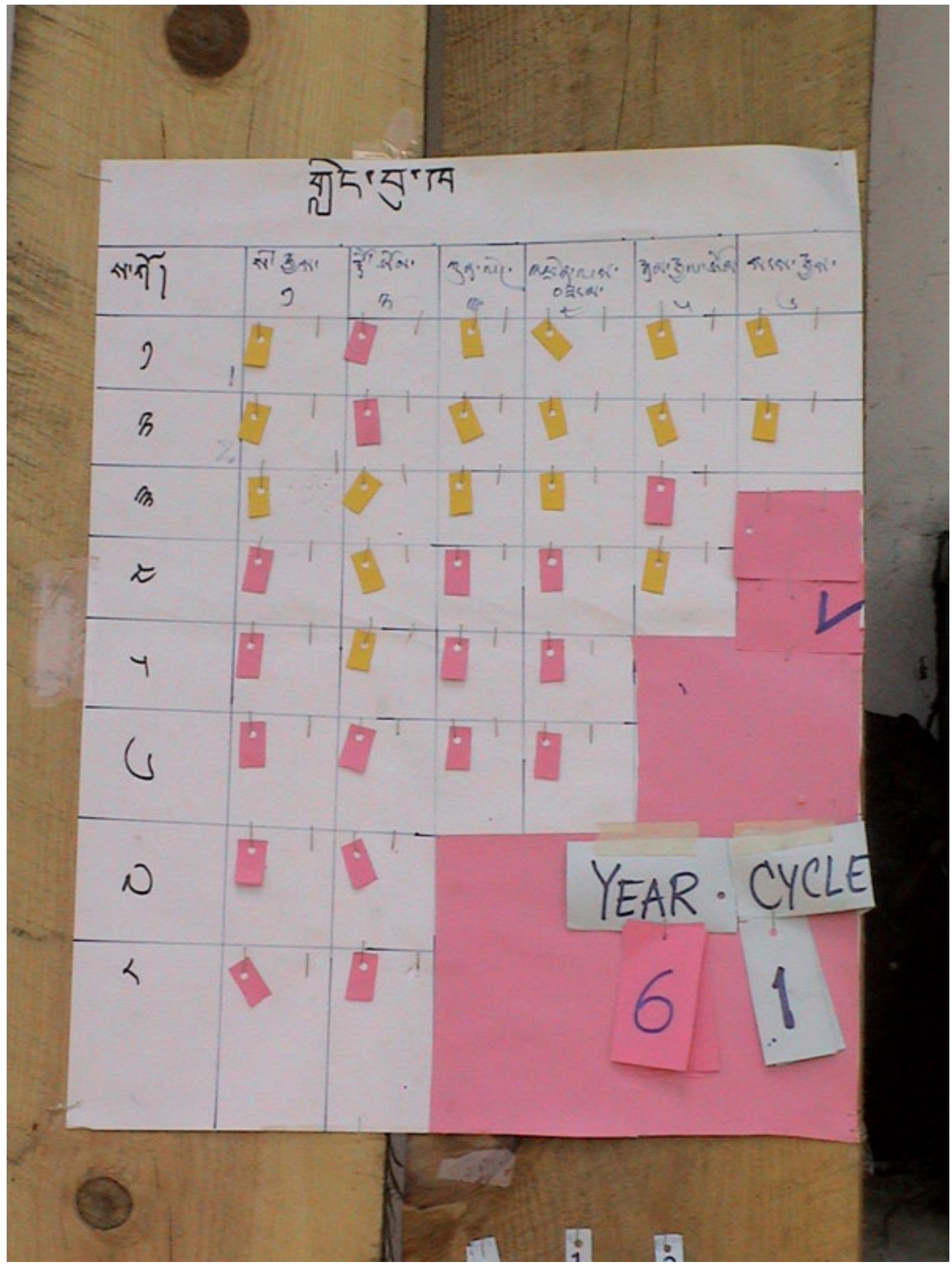


and observations, semi-structured individual interviews with each player were conducted to collect views on the game and evaluate it. Following the individual interviews, a plenary session was organized to present the preliminary results of each RPG session to the players and to get their response to the proposed analysis in the form of simple graphs of the land-use dynamics, water exchanges, and income.

\section{Results}

Knowledge representation and its validation by the players

Most of the farmers considered that the gaming parameters represented the real situation. One farmer remarked, "It appeared like playing a game, but recalling in the evening all appeared precisely real and stimulating." The players adjusted themselves to the gaming environment after one round of play. Eighty-two percent of the respondents confirmed that the game board represented the distribution of their fields. During the intra-village communication mode, definite patterns existed in choosing crops and fields in the first cycle of each crop year. All accepted the categorization of farmers in terms of access to water and number of fields. But $27 \%$ (one each from Thruelpa, Cheep, and Chatro) of them thought that the cash allocation was too high because, in real life, farmers may not be in a position to gain access to that amount to start farming.

Water share, water units, and the influence of rainfall on water availability were the main features that players related to reality. Although water exchange depends on the demand from those who need it, kinship played a dominating role in the exchange of water. Whenever there was unused irrigation water, it was first given free of charge to relatives who needed water. It was stated that it is shared on the basis of helping each other in times of need. Only after satisfying the demand of relatives would they trade with other players wanting to exchange labor for water. In the first gaming session, players introduced exchange of water for cash. Initially it was assumed that potato cultivation in Limbukha would affect access to irrigation water by Dompola farmers. Players said that potatoes are in fact harvested before the rice transplanting season starts in Dompola. Therefore, potato crops planted in Limbukha terraces did not influence Dompola's water share.

Of the three scenarios, farmers preferred the second scenario because it allowed them to collectively share resources and work together, which does not happen in reality. One participating member stated, "it is more fun and interesting to work together in a community, helping each other to pull along." Players further said that they were of the opinion that the existing water-sharing system was sound and two villages could never work together due to the physical distance between them. The second scenario allowed players to exchange water against labor between two villages. Although this exchange of water between the two villages does not exist in reality, $45 \%$ of the players responded that water exchange could happen between the two villages. Further, they suggested that, when there is plenty of water at the source, it should be shared. Given the increased dependence of Limbukha on farm labor from other villages and other socioeconomic factors, this should provide a basis for cooperation and a collective decision-making process in natural resource management, primarily for water.

\section{Learning}

As a learning experience from the game, $36 \%$ of the players reported that it helped them to understand the benefits of sharing water with neighbors both within and between two villages, to enhance their land-use system, productivity, and income. The game also helped $27 \%$ of the respondents understand the valuation of water share. This implied that, given the opportunity, a water market could emerge in the system.

Apart from the economic valuation of water, the game helped open up new understanding of the social dependence between villages, particularly in terms of labor in exchange for water and other services. The players also believed that the RPG helped them understand the value of maintaining farm accounts, the problems of a neighboring village, and the importance of completing farm work on time. For Dompola farmers, the game gave them the idea to attempt cultivation of a potato cash crop to increase their incomes either in Dompola or by leasing land in Limbukha where soils are more suitable for growing potato. 
Comparison of the lessons learned from the two gaming sessions held in May and December 2003 indicates that, over the period between two sessions of RPG, community members informally discussed and even assessed the impact of their decisions on resource sharing. It was unfortunate that these discussions could not be observed and recorded. A player from Limbukha said that they had discussions on water sharing before attending the second RPG session. In both cases, importance of sharing water was the most important lesson for all players. Compared with the lessons learned in May $2003,90 \%$ of the players in December 2003 learned of the need and benefit of water management and sharing $(70 \%$ water sharing, $10 \%$ canal management, and $10 \%$ on-farm water management; Fig. 4). This shared learning is an important output from RPG and it is expected that it will have dramatic influence on the way players will behave in future.

\section{Lessons from the Workshop}

The farmers of two conflicting villages willingly accepted the RPG as a means of expressing their concern about water sharing. The results from the game indicated that the RPG has been effective for collective learning, learning about the problem and process. The game outputs fostered better understanding of the problem of water sharing and its impact. The use of three scenarios (modes of communication) created a friendly environment for active participation of the players.

From the game, it was clear that rainfall is a determining factor in ensuring the availability of irrigation. A kinship network determines how irrigation water is shared within a village. This closed sharing system is assumed to be a risk avoidance strategy when resources are limited. Within each village, players exchanged water for labor or cash. The importance placed on water is demonstrated by the structured and fixed watersharing system followed by Limbukha village. Dompola lacked a structured system for sharing water, resulting in theft of water resources and time spent on guarding the canal. The opportunistic behavior of Dompola farmers could be related to an unstable (uncertain) irrigation supply. The game also revealed that the alternative communication mode can provide many opportunities for players to test its applicability. Unused irrigation water in
Limbukha was efficiently exchanged for surplus labor available from the village of Dompola. It was also clear that assigning a monetary value to water makes players more judicious in their use of water. As the intra-village communication mode represented the reality, players tended to perform better even in the game. The inter-village communication mode did not influence Limbukha players in terms of resource use and income. However, it was clear that, in the collective mode, Limbukha players could share all the unused irrigation water with Dompola players. Over all, Dompola players benefited more from the collective communication mode.

\section{Computerized Simulations}

The information generated by the diagnostic study and the RPGs was used to implement the computerized MAS model. Following the RPGs in May and December 2003, a MAS model was developed that was called the "Limbukha model." The objective of the model was to simulate various scenarios. The entities were identified and an initial class diagram was constructed to show all the model entities, attributes, and methods, and their structural relationships (Fig. 5) ${ }^{[2]}$.

The model is described in Append. 2.

\section{Test of the model}

The scenario in which players first gave water to their kin, followed by trading with acquaintances was used for the test run because it is the most realistic one. As Manson (2002) suggested, scenarios have been examined from a number of stylized, theoretical perspectives to see if they are qualitatively reasonable. Similarly Bousquet et al. (2002) also indicated that the validation of models could be partly done by interviewing experts. Thirty simulations of the base model were run to check its consistency and behavior. Only thirty simuations were run because the objective here is not to propose a sensitivity analysis. Each simulation was run over 20 time steps. The outputs of the simulations were captured just to test its consistency. Excel ${ }^{\circledR}$ spreadsheets and several graphs were generated. As the RPGs were played only over six time steps, it is not possible to compare the RPGs and the MAS outputs. Visual comparison of model outputs with those of the RPGs was done to assess consistency. 
Fig. 4. Lessons learned by players from the two gaming sessions in May and December of 2003.

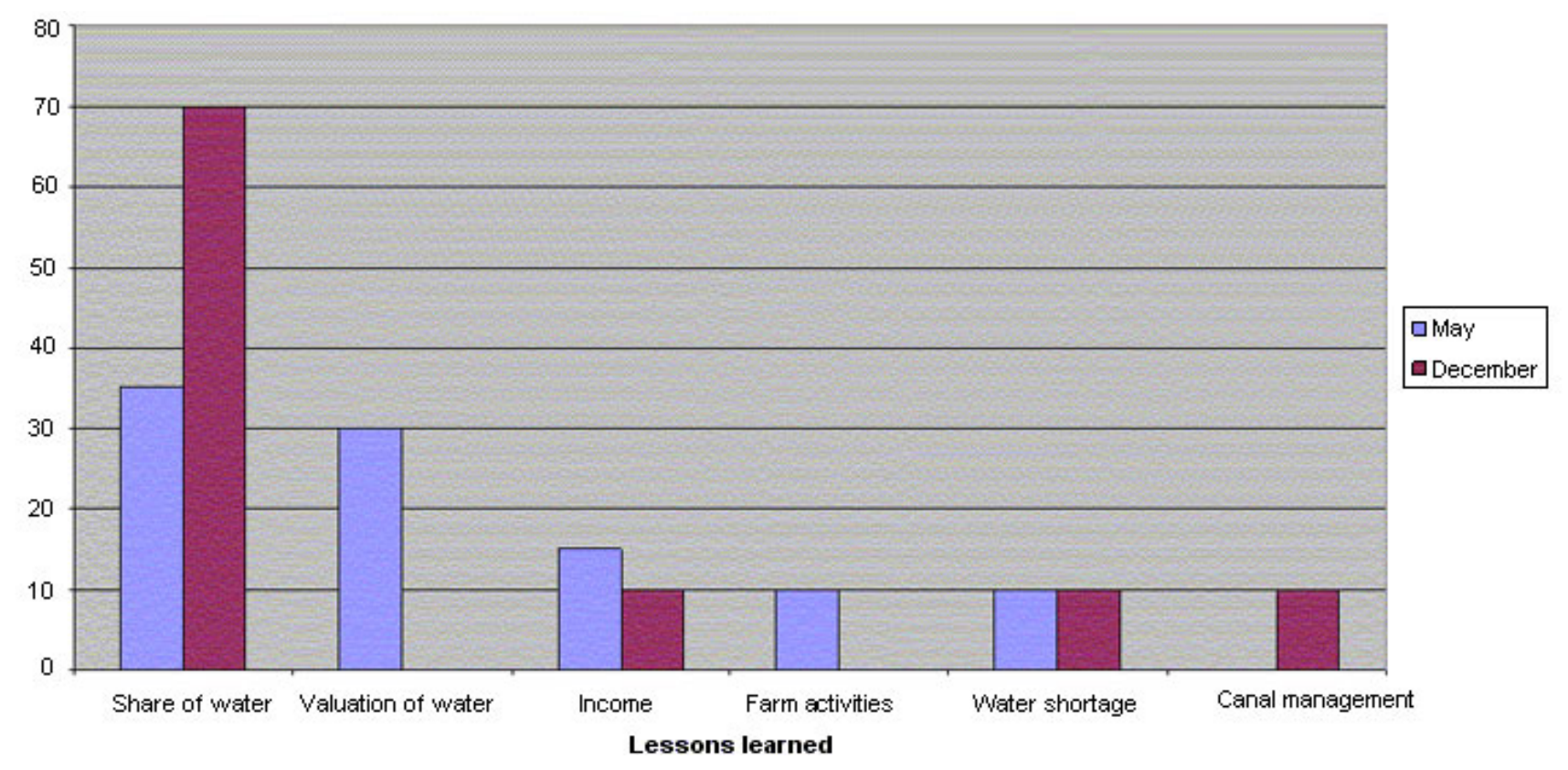

The simulation outputs shown in Fig. 6 indicate the similarity between the base model and RPG outputs. At least they react consistently to changes in parameters. For instance, the number of plots planted with rice (Fig. 6a) consistently remained within the range of $60 \%$ to $90 \%$, varying according to rainfall pattern and market state. This corresponds to the sum of rice plots in a year for the two villages in the Dompola RPG. The number of plots planted with potato in Fig. $6 \mathrm{~b}$ behaved differently from the RPG output. The main difference was the absence of potato in some years in the model output, whereas the RPG results show potato being grown every year in Limbukha. The reason for not planting potato was a result of the condition of market price and rainfall pattern used in making the cropping pattern decision in the MAS model. A peculiar behavior of the model was that potato plots varied between $0 \%$ and $25 \%$, indicating that there could be some weakness in the model compared with the RPG. In any case, it maintained the maximum limit of 17 plots. The amount of unused water units in the model fluctuated between 4 and 16, depending on rainfall pattern (Fig. 6c). It appeared that the model overestimates the amount of unused water compared with the RPG output, where the maximum number of units of unused water was six. This could be due to the protocol, which has to be strictly followed when exchanging water in the model. From the way the model behaves, it is considered that it is consistent in terms of its response to the parameters used in the simulations.

\section{Scenarios}

As the Limbukha model was roughly able to represent the RPG, it was used to generate scenarios to understand the potential effects of changes in strategies on the resource and the economic returns of irrigators. To generate multiple scenarios, three main parameters (namely, social networks, rainfall patterns, and exchange protocols) were identified (Table 2). Accordingly, 36 scenarios with 20 runs per scenario were produced. Data from each scenario were captured in Excel spreadsheets.

\section{Indicators}

The 36 scenarios were further classified based on their fulfilment of six criteria (Table 3). The classification was necessarily used to categorize and identify potentially viable scenarios. Thresholds for 
Fig. 5. Class diagram of the Limbukha model.

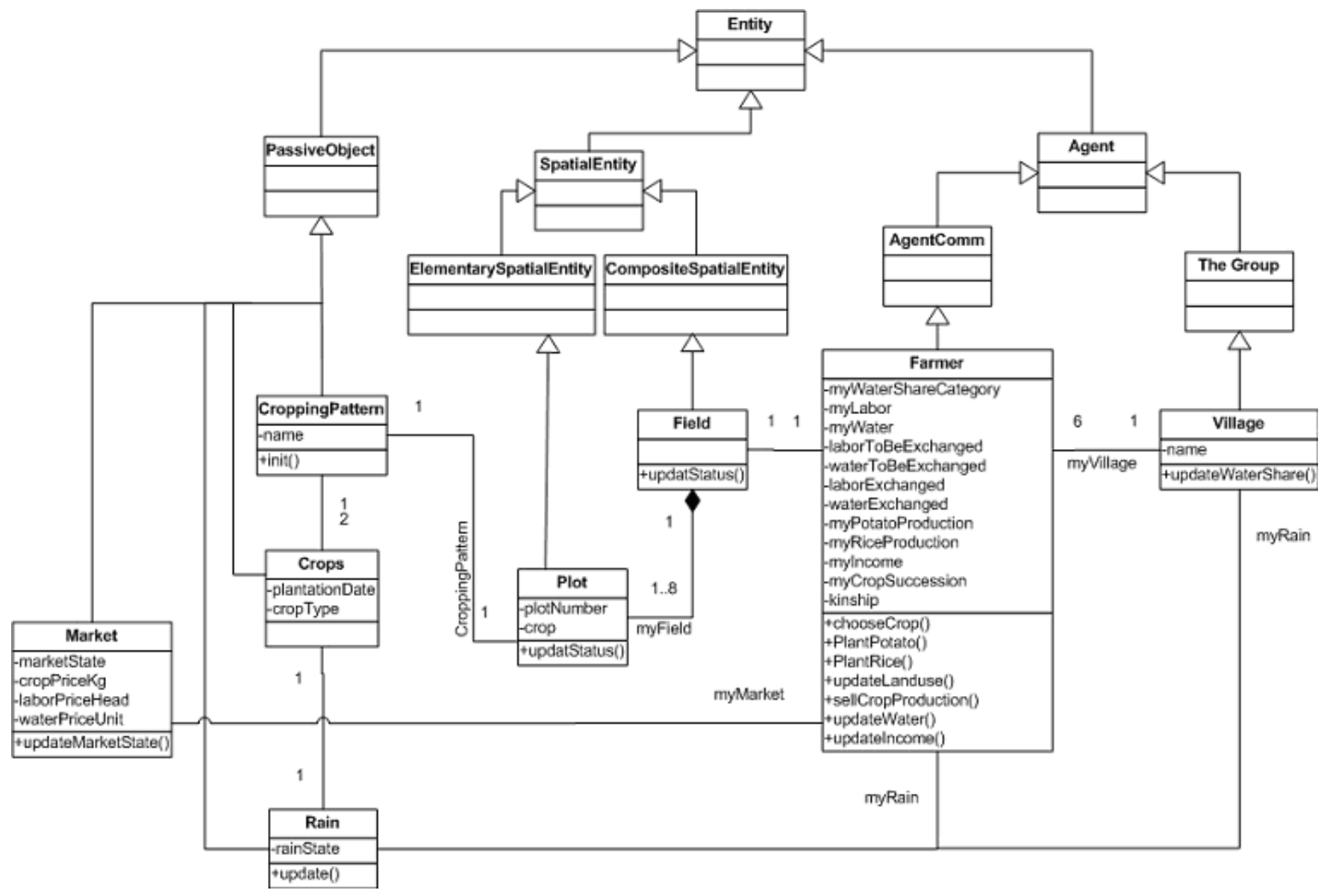

each indicator were based on the researcher's perception of the situation, e.g., the minimum number of plots planted to rice should be 12; fallow plots should not be more than seven; there should be at least six potato plots; there should be at least one instance of exchange of unused water, which should total less than three units; and finally, total annual income should be more than US\$10 000 .

Three measures of viability (high, medium, and low) were used to assess the scenarios for further exploration. A scenario was considered highly viable if it fulfilled more than five criteria and conditions displayed in Table 3. Similarly, they were categorized as medium if they fulfilled only 3-4 criteria, and low if they fulfilled 2 or less criteria.
The results summarized in Table 4 show that $71 \%$ of the scenarios displayed a medium viability. Most scenarios based on interactions among kinship are only of medium viability, and there are no highly viable scenarios. Interactions among kinship and acquaintances within and between villages resulted in $6 \%$ and $8 \%$, respectively, of the scenarios fulfilling more than five criteria. It further validates the finding of the RPG that a collective communication mode facilitates better resource use and also fulfills other socioeconomic objectives. Tables 5, 6, and 7 indicate that the viability of the scenario can be greatly influenced by the communication protocol, and that rainfall pattern does not have much influence. 
Fig. 6. Test of the Limbukha base model indicating (a) rice plots, (b) potato plots, (c) unused irrigation water, generated from 30 simulations of the base model.

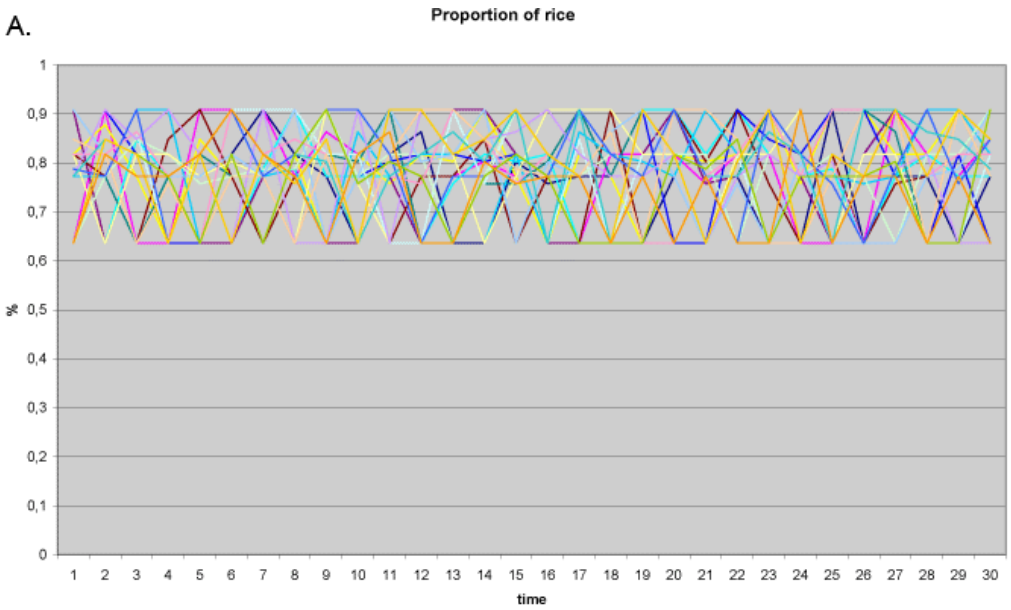

B.

Proportion of potato

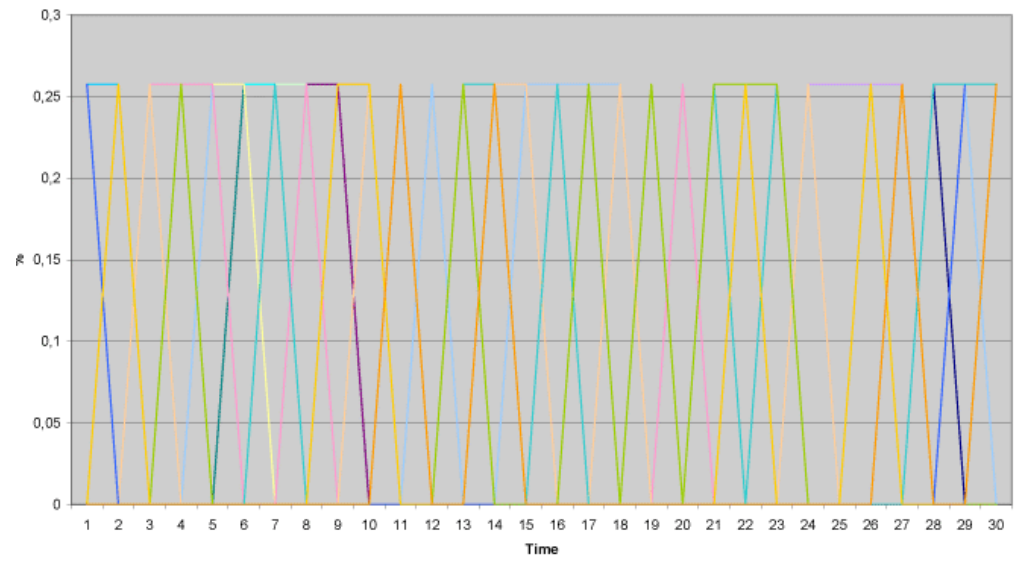

C.

Unused water

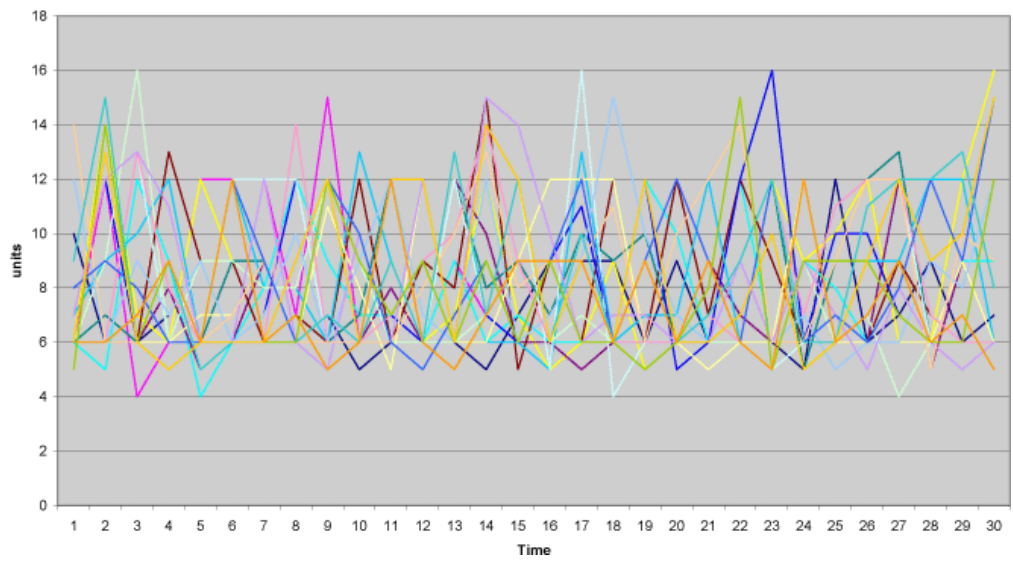


Table 2. Possible factors to be simulated with the Limbukha model

\begin{tabular}{ll}
\hline \hline Parameters & Variables \\
\hline Social network & $\mathrm{N}_{1}$ : Kinship \\
& $\mathrm{N}_{2}$ : All members of same village (N1 + acquaintances in the same \\
village) \\
$\mathrm{N}_{3}$ : Members of both the villages $(\mathrm{N} 1+$ acquaintances in both villages) \\
& $\mathrm{R}_{1}$ : Predominantly Low \\
& $\mathrm{R}_{2}$ : Predominantly High \\
& $\mathrm{P}_{1}$ : Give water to kinfolk \\
& $\mathrm{P}_{2}:$ Exchange water for labor or cash \\
& $\mathrm{P}_{3}:$ P1 + Exchange labor for cash \\
& $\mathrm{P}_{4}:$ Exchange water free of charge \\
& $\mathrm{P}_{5}:$ Exchange labor for water \\
& $\mathrm{P}_{6}: \mathrm{P}_{1}+\mathrm{P}_{2}+\mathrm{P}_{5}$ \\
& \\
\hline
\end{tabular}

\section{Discussion on the RPG and Computerized Model}

Some of the critical findings of the two RPGs and the MAS model are that: (i) the RPG effectively facilitated self-motivating and non-confrontational interactions among the players; (ii) farmers' knowledge and understanding of water sharing increased significantly between two RPGs; (iii) exchange protocols influenced water use and income more than the rainfall pattern and social networks; and (iv) the collective mode of communication facilitated better and more frequent exchange of water.

The role of the computerized model was to explore scenarios that were collectively identified during the RPG sessions by the stakeholders. Three dimensions were explored, the natural climate variability, the social networks, and the exchange protocols. It appears from these simulations that the critical factor is the exchange protocols. Together with the field observations, the lesson of these simulations is that, in the field, improvement depends on defining better communication protocols. Concretely, in the field, the next step was to set up a communication platform to define these protocols.

The December RPG was remarkable in the history of the Lingmutey Chu watershed. It can be considered as a breakthrough in the mediation process of developing an efficient water-sharing system. The two noteworthy proposals of the workshop were (i) Limbukha will release irrigation water 5 days earlier than the $10^{\text {th }}$ day of the $5^{\text {th }}$ month of the lunar calender, and (ii) there is a need to establish a management committee at the watershed level to promote and oversee the watershed management activities. The year 2003 came to an end with a high expectation of people being reasonable and sharing whatever they have. However, in May 2004, the Dompola farmers approached the people of Limbukha to discuss changing the water release date. To everyone's surprise, the Limbukha Tsogpa, although acknowledging that the issue had been discussed and resolved, claimed a legal agreement should have been signed following the resolution in December (nothing was signed at that time). The news spread fast, and RNRRC Bajo, as the facilitating agency, also attempted to convince the Limbukha Tsogpa, but he stood firm in his claims. Thus, the traditional system prevailed and the Limbukha farmers continued to exercise their traditional rights over water. This means that the workshops in April and December 2003 were taken very seriously and that a formal agreement should have been signed after the workshops. The process went further and faster than the facilitators of the process expected.

However, this denial was the principal lesson for the researchers and for the people themselves. Following the recommendations of the 2003 RPGs, 
Table 3. Classification of scenarios based on thresholds of six criteria and conditions. First digit represents social network $(1,2,3)$; second digit represents rainfall (1 and 2); and third digit represents protocol (1 to 6)

\begin{tabular}{|c|c|c|c|c|c|}
\hline $\begin{array}{l}\text { Unused irrigation } \\
\text { water } \\
(<3 \text { units })\end{array}$ & $\begin{array}{l}\text { Fallow plots } \\
(<7 \text { plots })\end{array}$ & $\begin{array}{l}\text { Potato plots } \\
(>6 \text { plots })\end{array}$ & $\begin{array}{l}\text { Rice plots } \\
(>12 \text { plots })\end{array}$ & $\begin{array}{l}\text { Annual income } \\
(>U S \$ 10000)\end{array}$ & $\begin{array}{l}\text { Water exchange } \\
\text { (>1 transaction) }\end{array}$ \\
\hline $\begin{array}{l}112,113,122, \\
123,213,215, \\
216,222,223, \\
224,225,226, \\
313,315,316, \\
322,323,324, \\
325,326\end{array}$ & $\begin{array}{l}111,114,115, \\
116,124,126, \\
213,214,223, \\
226,311,313, \\
323\end{array}$ & $\begin{array}{l}112,113,114,115, \\
116,121,122,125, \\
126,211,212,213, \\
214,215,221,222, \\
223,224,225,311, \\
312,313,314,315, \\
321,322,323,324, \\
325,326\end{array}$ & $\begin{array}{l}\text { All Scenarios } \\
\text { (as minimum } \\
\text { number of } \\
\text { plot is } 43 \text { ) }\end{array}$ & $\begin{array}{l}111,114,115,116, \\
121,124,125,211, \\
212,213,214,223, \\
226,311,312,313, \\
314,321,322\end{array}$ & $\begin{array}{l}212,222,312,313, \\
314,322,323\end{array}$ \\
\hline
\end{tabular}

the RNRRC planned yet another RPG called the "Seven Villages Game" for 2005. The game was intended for the seven villages ${ }^{[3]}$ of the Lingmutey Chu watershed.

\section{Collective Workshop to Establish a Watershed Resource Management Committee}

The objectives were to:

- facilitate exchanges among the seven villages regarding NRM at the watershed level,

- enhance the stakeholders' understanding of the resource use dynamics at the watershed level, and

- define the next steps toward the establishment of a formal watershed management committee (WMC) before late 2005, and plan several short-term priority actions in INRM.

The RNRRC researchers prepared a new RPG and organized a workshop. The RPG and the workshop settings are described in Append. 3.

\section{RPG Scenarios}

The RPG was designed in three communication modes, to provide different settings for players to promote communication and generate new ideas to cope with the emerging situation.

\section{First scenario}

As far as possible, the design of the first RPG was maintained to represent the reality. In a real-life situation, irrespective of their turn and corresponding to the rice transplanting, farmers start transplanting as soon as they access sufficient irrigation water. It is assumed that water-sharing rules within the village are vital and that the emergence of the interaction among the players within a village determined the availability and flow trend of excess water. The scenario was designed with the objectives defined as follows:

1. To allow players to familiarize themselves with the gaming principles which, to a large extent, represent reality.

2. To identify sharing mechanisms within a village.

The rules of the first RPG were simple and straightforward. They can be simply described as follows: 
Table 4. Proportion (\%) of scenarios under different levels of viability based on three types of social networks

\begin{tabular}{|c|c|c|c|c|}
\hline \multirow[t]{2}{*}{ Network } & \multicolumn{3}{|c|}{ Viability of scenario* } & \multirow[b]{2}{*}{ Tota } \\
\hline & High & Medium & Low & \\
\hline Kinship & 0 & 27 & 6 & 33 \\
\hline Kinship and acquaintances within village & 6 & 22 & 6 & 34 \\
\hline Kinship and acquaintances of two villages & 8 & 22 & 3 & 33 \\
\hline Total & 14 & 71 & 15 & \\
\hline
\end{tabular}

$*$ High $=$ satisfies 5 indicators; Medium = satisfies 3 to 4 indicators; and Low $=$ satisfies less than 2 indicators

- Discussion is allowed only within the village.

- No member of a village is supposed to know about the actions of the other village.

- After the water share for each village is declared and allocated, each member of a village should be involved in discussions to share water among themselves.

- Any remaining (surplus or unused) water should be left in the rectangular scoop.

In sequence, these were the various steps:

Set-up:

1. The objective of the game and its origin was explained to all the participants. The rules of the game were explained thoroughly and even demonstrated. Each component of the game was explained thoroughly. All players were introduced. However, it was left up to each group to develop and adopt their own strategies.

2. Each village (Irrigator) was given its own game board (block of fields and plots assigned).
3. Players (Management group) were assigned their tasks.

4. Players (Market) take their position with computer and cash.

5. Facilitators take their group to an assigned place for the game.

Step 1: The facilitators of the game (Fig. 7 a)

1. Declare the date (in weeks ${ }^{[4]}$ ) of release of water.

2. Draw and declare weather (Severe wet/Wet/ Moderately dry/Dry).

3. Based on the weather, declare and allocate water to each group.

Step 2:

1. Players from each community discuss strategy among themselves (not letting the other community know) and plant the riceby placing the dice representing water. Players can place a maximum of 2 units of water in a plot (scoop) in the game board (Fig. 7b). 
Table 5. Number of scenarios under different levels of viability based on six types of protocol

\begin{tabular}{lcccc}
\hline \hline Network & \multicolumn{5}{c}{ Viability of scenario* $^{*}$} \\
\hline P1 & High & Medium & Low & Total \\
P2 & 0 & 5 & 1 & 6 \\
P3 & 1 & 5 & 0 & 6 \\
P4 & 4 & 1 & 1 & 6 \\
P5 & 0 & 6 & 0 & 6 \\
P6 & 0 & 6 & 0 & 6 \\
Total & 0 & 3 & 3 & 6 \\
& 5 & 26 & 5 & \\
* High = satisfies 5 indicators; Medium = satisfies 3 to 4 indicators; and Low = satisfies less \\
than 2 indicators
\end{tabular}

2. In the case of any excess water, players decide what to do (options are: use it themselves/let it accumulate/share it with a neighboring community; these options were not declared).

3. The facilitator records the processdiscussion on how they decided what they did (Fig. 7d).

4. The facilitator records the number of dice in each plot, using a data recording sheet.

5. The sheet is then passed to the Market, where the data are entered in an Excel spreadsheet.

6. Individual players are called by the Market group and paid their income (Fig. 7c).

7. The organizers assess the environmental impact and declare the impact at watershed level; they also declare how many villages performed negatively. They give a colored card as a reward to the Management group.

8. The facilitator collects all the dice; return to Step 1 for next round.

\section{Second scenario}

The second RPG was played on the same day in the afternoon. It was played in a collective mode where each group stayed in close proximity in same order as in the watershed (Fig. 8). The scenario was played with the following objectives:

- To let players experience the collective mode.

- To promote collective decisions in irrigation water use and sharing.

- To encourage players to try different strategies of collective management, while sustaining or improving performance.

Although the basic structure of the game was same as in the first RPG, the rules specific to this game are:

- Players within a village first discuss and share available water.

- Depending on the water situation, they can confer with the neighboring village. 
Table 6. Number of scenarios under different levels of viability based on six types of protocol

\begin{tabular}{lcccc}
\hline \hline \multicolumn{1}{c}{ Network } & \multicolumn{5}{c}{ Viability of scenario* $^{*}$} \\
\hline P1 & High & Medium & Low & Total \\
P2 & 0 & 5 & 1 & 6 \\
P3 & 1 & 5 & 0 & 6 \\
P4 & 4 & 1 & 1 & 6 \\
P5 & 0 & 6 & 0 & 6 \\
P6 & 0 & 6 & 0 & 6 \\
Total & 0 & 3 & 3 & 6 \\
& 5 & 26 & 5 & \\
* High = satisfies 5 indicators; Medium = satisfies 3 to 4 indicators; and Low = satisfies less \\
than 2 indicators
\end{tabular}

- Players are allowed to move around to observe what others are doing (free discussion).

- Any remaining (unused) water will flow down to the river (in this case, one of the facilitators collected the remaining cube [water shares] and placed it on the last board, declaring that a given number of water units was drained into the Puna-tshang chu).

Set-up and Step 1: (same as in RPG 1).

Step 2: (In addition to steps as described in RPG 1):

1. Players discuss among themselves and plant the rice, by placing the dice representing water. They can move between groups either to offer what they have or to share the balance they have.

2. In the case of any excess water, players decide what to do (options are: use it themselves/let it accumulate/share it with the neighboring community; these options were not declared).
3. The facilitator records the process, and a discussion ensues on how they decided what they did.

4. The facilitator collects all the dice; return to Step 1 for next round.

\section{Third scenario}

The third RPG was played in a swapped mode, where players changed their roles. Given that players have been exposed to the individual and collective modes of the game in the earlier games, this game emphasizes putting the player in different situations, such that innovative realization emerges from the game. The specific objectives of the scenario can be summarized as follows:

- To provide players with the opportunity to play the same game, but from another player's perspective.

- To explore and observe novel processes and interactions. 
Table 7. Number of scenarios under different levels of viability based on two types of rainfall

\begin{tabular}{lcccc}
\hline \hline Rainfall & \multicolumn{4}{c}{ Viability of scenario $^{*}$} \\
\hline \multirow{2}{*}{ High } & Medium & Low & Total \\
1 & 2 & 14 & 2 & 18 \\
2 & 3 & 12 & 3 & 18 \\
Total & 5 & 26 & 5 &
\end{tabular}

* High $=$ satisfies 5 indicators; Medium $=$ satisfies 3 to 4 indicators; and Low = satisfies less than 2 indicators

Specific rules

As the main purpose of this scenario was to provoke closer interaction among the players, the game was set up in a very compact setting. In addition to the basic rules of the game followed in other scenarios, some specific rules are:

- Each player is randomly placed in a group of three, representing a random village.

- Players within a village are allowed to confer with any players.

- Any player can intervene in the decision making and seek an explanation.

- Collective decision making (consensus) is honored, rather than an individual's decision.

- Players are allowed to move around to observe what others are doing (free discussion).

The third scenario was initially designed to be done with swapped roles, mainly players from one village, as a group, would be randomly assigned to a new village to play in individual communication mode as in the first RPG. However, seeing the progress made in the collective mode and the need for more interactions among the players, facilitators discussed and introduced the following change. In the new format, players were randomly numbered 1 to 7 , and all those with the same number (e.g., all $1 \mathrm{~s})$ formed a group of three. These groups drew to identify which village they represented. In order to achieve greater mobility of players, better visibility of the game board, and closer interaction among the players, the game boards were arranged on a table in the same sequence as the villages in the watershed (Fig. 9).

As the weather was declared by game organizer, group facilitators placed a designated amount of water share on the game board (Table 8), and the players began playing. Unlike the previous games, interactions among players across the group were self-motivated and productive. Every move by a group was closely monitored by other members and any new moves (strategy) were somehow made public as players pressed the group to provide an explanation for their actions. Observations and comments were spontaneous and critical. It was anticipated that swapping positions would encourage commentary as, released from the restrictions of their real situations, they did not risk hurting anyone's feelings. Although each round of the third scenario took more than 20 to 30 minutes to play, it was encouraging to see the quality of interactions among the players.

After each round, players were paid their income as in the other games. To encourage collective decision making, and to demonstrate the impact of such actions, a parameter of environmental impact 
Fig. 7. Clockwise from upper left: declaring the weather and watershare, game in session, marketplace (settling accounts), and interview with player following the RPG.
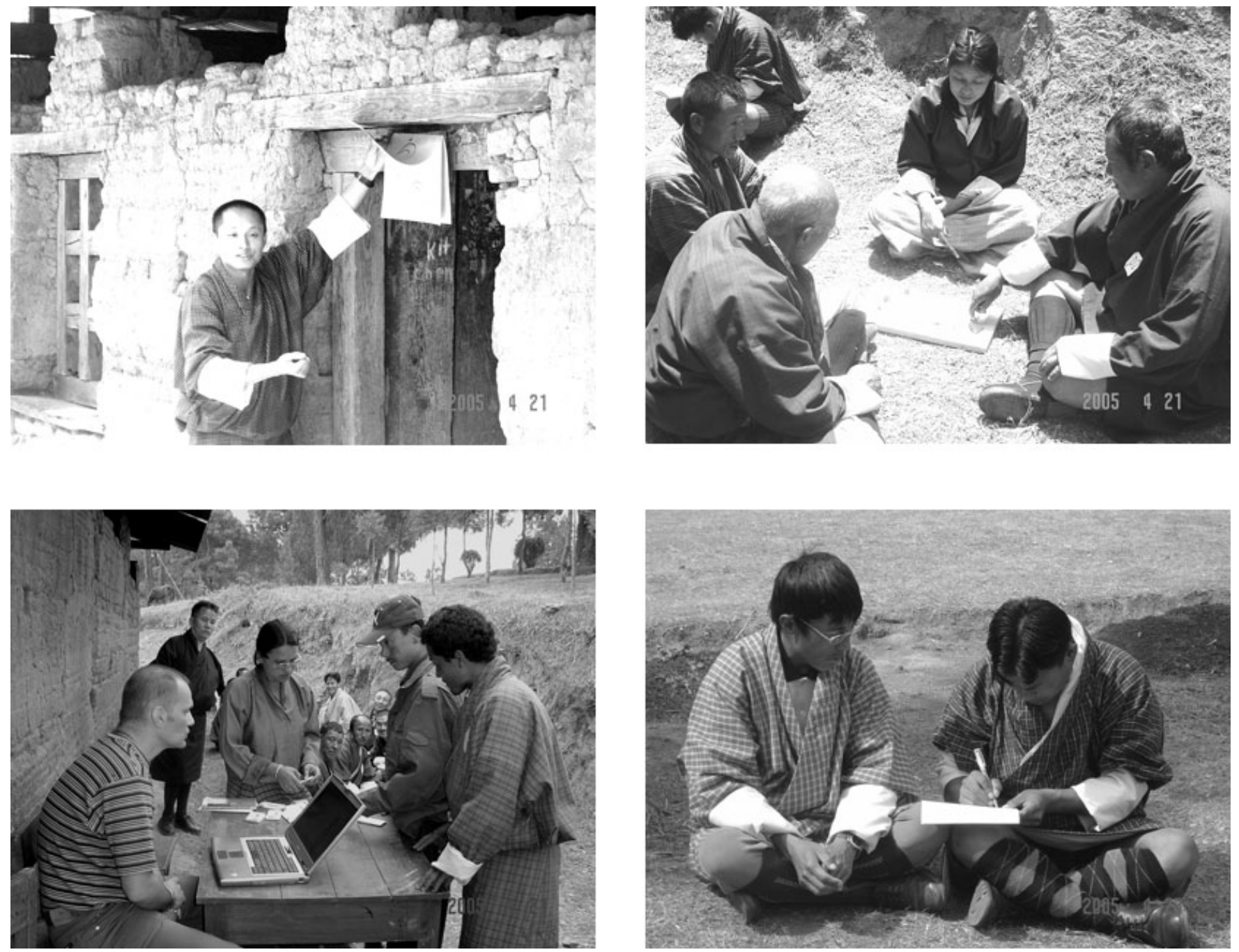

assessment was built in the game. The theory of reward for better water management (equating to efficient on-farm water use, sharing and saving strategies) was included in the game, and was assessed after each round. Rewards depend on the amount of water used per plot at the individual level; thereafter, impact is consolidated at the watershed level (Table 9). Based on the impact at the watershed level, rewards are determined by the game facilitator at the end of the round (Fig. 10). For simplicity, one unit of reward equals NU. 1000, and reward units were handed over to Mangmi (the Deputy Village Headman) after each round (Table 9).

\section{RPG Results}

\section{Water sharing}

Figure 11 clearly shows that the individual mode of communication did not promote water sharing. Similarly, during the dry season (lowest rainfall), there was no sharing reported. In the game, water sharing was recorded in the collective and swapped collective modes in three rainfall patterns but not in the dry season. Maximum sharing was found when rainfall was heavy and in the swapped collective communication mode. This was expected, as 
Fig. 8. Second RPG in session.

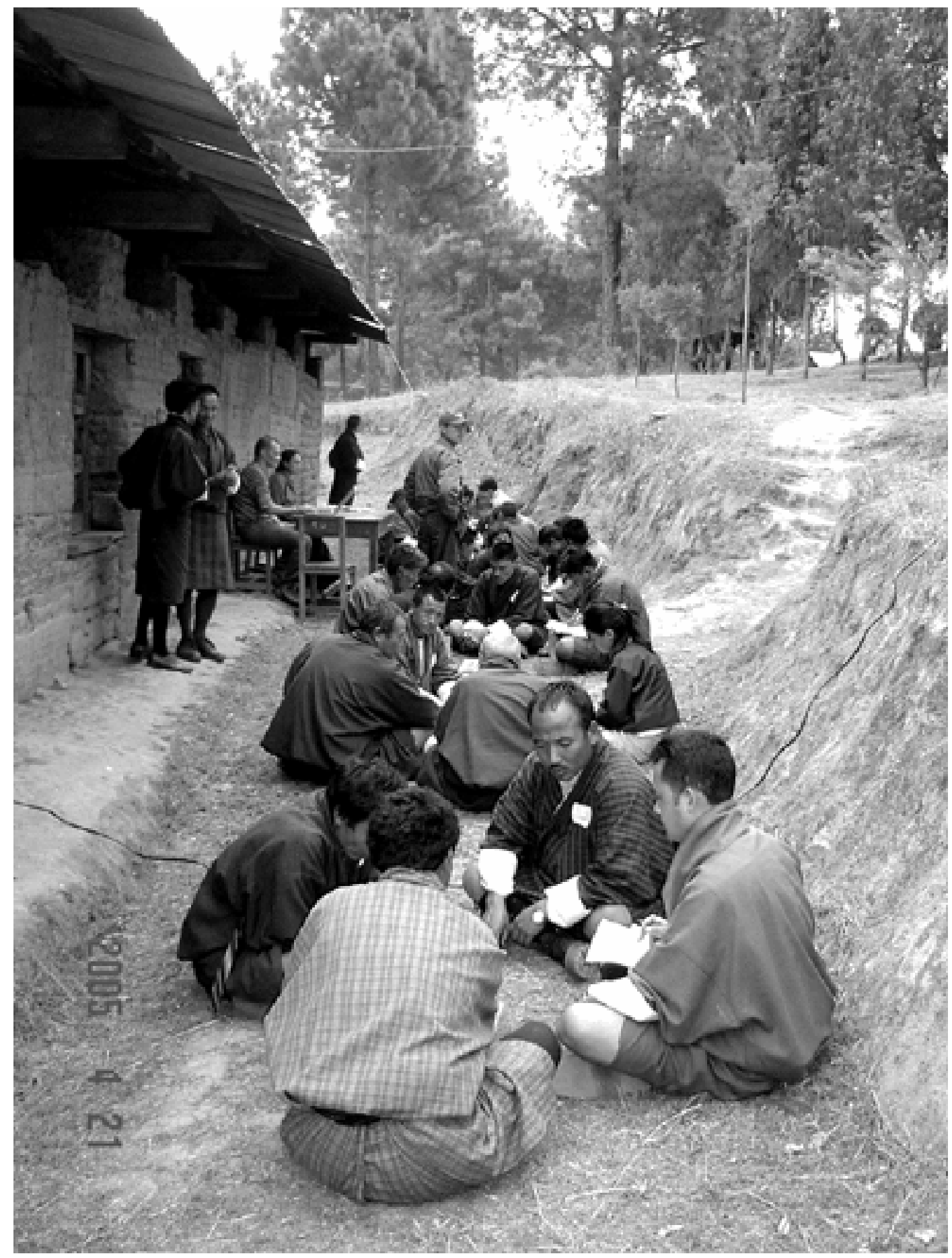


Fig. 9. Third RPG in session.

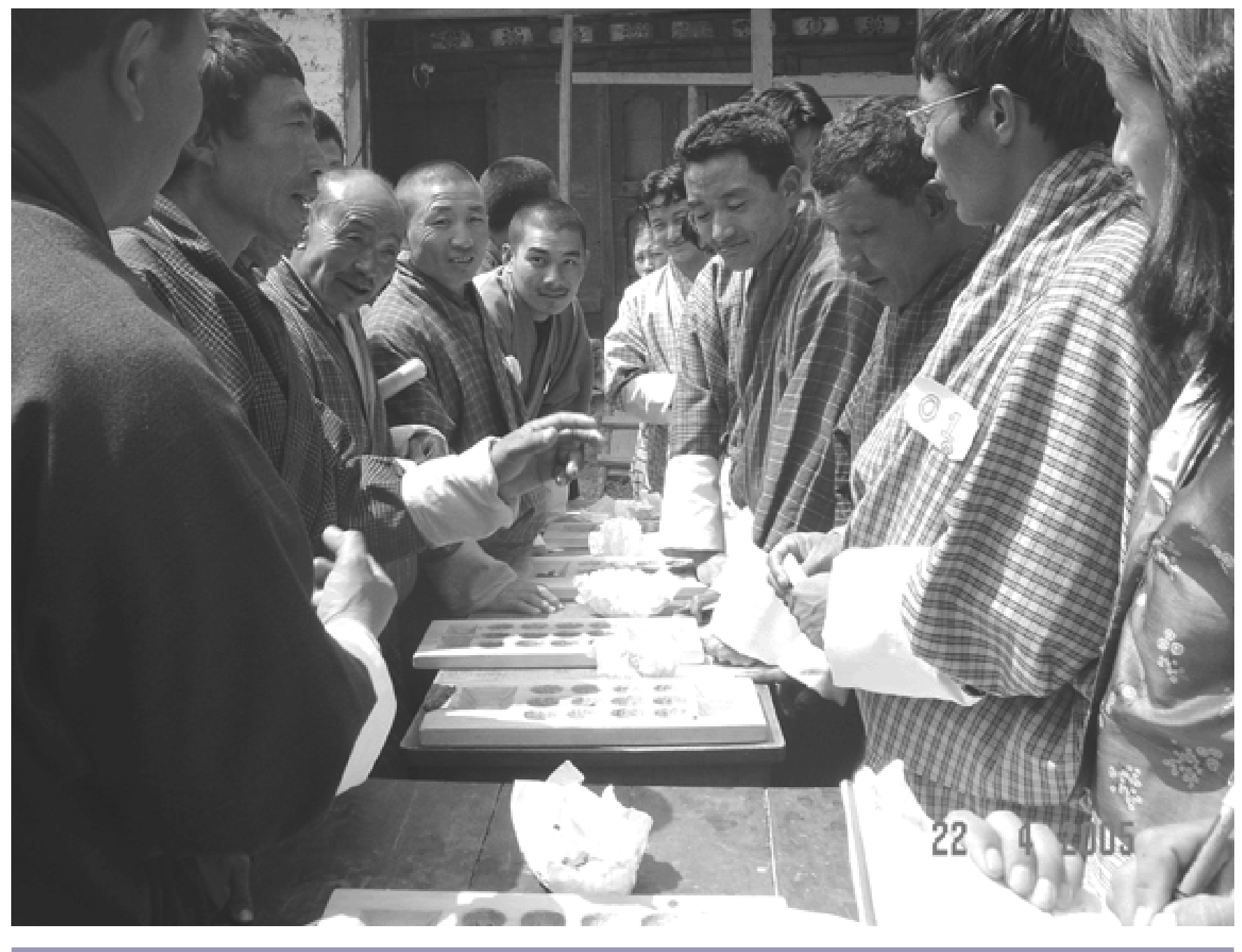

players in swapped roles had more opportunity to interact and bargain for the excess water. Considering the interactive communication in the swapped mode, both bargaining power and the exchange process were facilitated.

\section{Income}

After every game, the players were paid the income earned by cropping their plots using their water share. In all three communication modes, Limbukha village generated on average $34 \%$ more income than the other villages. The highest income difference of $>80 \%$ was observed between Limbukha and
Dompola villages in the individual and collective modes. Interestingly, Dompola's income in the swapped collective mode increased by $37 \%$ compared with the other two communication modes. Although there was no distinct influence of communication mode on Limbukha's income, the collective mode helped raise income in Nabchee, Omteykha, Matalumchu, Wanjokha, and Thagu by $8 \%$ to $14 \%$ (Fig. 12). Overall, the collective mode of communication, which promoted free exchange of goods and services, led to higher incomes in most villages. 
Table 8. Water share per village

\begin{tabular}{lccccccc}
\hline \hline Weather & \multicolumn{7}{c}{ Village } \\
\hline & 1 & 2 & 3 & 4 & 5 & 6 & 7 \\
Severe Wet & 25 & 9 & 13 & 13 & 13 & 13 & 13 \\
Wet & 19 & 7 & 10 & 10 & 10 & 10 & 10 \\
Moderately Dry & 13 & 5 & 7 & 7 & 7 & 7 & 7 \\
Dry & 7 & 3 & 4 & 4 & 4 & 4 & 4 \\
\end{tabular}

\section{The Discussions}

Plenary sessions were a central element of the whole process. Following the traditionally accepted custom of "Zomdu" (gathering for discussion) at all local levels, plenary discussions are considered appropriate. Most often, the decorum of these "Zomdu" adds to the formality of the process. Although monotonous sometimes, tremendous wisdom can be found in these long discourses and people feel structured and comfortable being part of such processes. In the case of the RPG organized in April 2005, a different form of plenary was used. Roughly three plenary sessions were conducted over the course of a day to (i) introduce and brief participants, (ii) present results or progress, and (iii) discuss critical points concerning watershed management. Whereas the facilitators discussed among themselves and worked late into the evenings, farmers had discussions in their respective villages late at night to inform the villages and get their opinions for the next day's session. This served to indicate the seriousness of the issue and their commitment to the common cause. Overall, plenary sessions formed part of the learning process for both participants and facilitators. They provided speedy analysis of the issues and faciliated envisionment of alternative pathways.

\section{The Agreement}

The outcome of the discussions regarding the need for and advantages and disadvantages of establishing a watershed institution-the Watershed
Management Group, Constitution and By-lawswere presented to the plenary session. The result of a secret ballot ranking preference for the scenarios indicated that the collective mode of RPG ranked the highest. This indicated that most of the participants favored working together toward a common vision of watershed development by establishing a watershed-level institution. To ensure nothing is left to chance, the floor also pledged (Fig. 13) to work toward the common benefit not only for the present generation, but also for many generations to come.

\section{First Actions Taken}

The work plan, which sets in motion the process of developing the bylaws (Table 10), was developed by the village "Tshogpas." The schedule aimed to complete the formulation of the bylaws by November 2005, and then present them at the plenary session for approval by consensus and formalization.

Besides forming committees and drafting the constitution and bylaws, workshop participants planned three collective actions that will be implemented immediately; these activities are:

1. Planting-Understanding the dynamics and relationships between forests and water, participants felt it necessary to protect springwater sources to improve ground water replenishment and volume of stream water. 
Table 9. Impact of water use and reward for better management

\begin{tabular}{|c|c|c|c|c|}
\hline $\begin{array}{l}\text { Water share units } \\
\text { used per plot }\end{array}$ & $\begin{array}{l}\text { Plot-level I- } \\
\text { mpact }\end{array}$ & $\begin{array}{l}\text { Village-level } \\
\text { impact }\end{array}$ & Watershed-level impact & $\begin{array}{l}\text { Reward } \\
\text { (REWARD units depend on the number of } \\
\text { "+" received at the watershed level) }\end{array}$ \\
\hline 0 & + & + & $\begin{array}{l}\text { If number of "+" } \\
\geq 4=\text { " +"" } \\
\leq 4=\text { ="” }\end{array}$ & $\begin{array}{l}4 “+”=1 \text { reward unit }(\mathrm{Nu} .1000) \\
5 “+"=2 \text { reward units }(\mathrm{Nu} .2000) \\
6 “+”=3 \text { reward units }(\mathrm{Nu} .3000) \\
7 \text { “+”= } 4 \text { reward units }(\mathrm{Nu} .4000)\end{array}$ \\
\hline 1 & + & + & & \\
\hline 2 & - & $\begin{array}{l}\geq 3="-" \\
\leq 3="+"\end{array}$ & & \\
\hline
\end{tabular}

Participants unanimously agreed to collectively plant tree saplings near water sources, but at the leve of individual villages.

2. Rehabilitation-During the workshop, there were presentations and discussion on how deforestation causes a decline in water volume. Farmers recognized that such a situation had occurred on the abandoned wetland at Lumpa, opposite Omteykha. Previously, it had been under cultivation but, over time, farmers to left the land fallow because of lack of water. The participants ensured that the abandoned land will be rehabilitated through collective action, which will be led by the village head (Gup).

3. Conservation-A shortage of irrigation water during rice transplanting was a major issue for the farmers of the Lingmuteychhu watershed. During the workshop and also during individual interviews, it was revealed that availability of irrigation water depends on the amount of rainfall. If there is a good rainfall, a good amount of water flows downstream to the fields and then to river below. To check this surface water run-off during the monsoon and to harvest it, participants agreed to construct small water reservoirs-like ponds, check dams, etc.—in each village, because they will help recharge the ground water and can be used during seasons of scarce water.

\section{Impact and Perspectives}

The impact of the April RPG was monitored over a period of 7 months. In parallel, a team of farmers and a facilitator (designated as the drafting committee) worked on developing the constitution and by-laws for the Lingmuteychu Watershed Management Committee. In November 2005, which corresponds to $7^{\text {th }}$ month after the April RPG, the draft constitution was presented to the community and the committee was formally instituted. A 3-day participatory workshop was organized to finalize the constitution and by-laws for the Watershed Management Committee. In the same workshop, a summary of the monitoring reports was presented. The Watershed Management Committee was formally established in the Lingmuteychu Watershed.

During the first half of 2006, the research team facilitated the design and preparation of a request for external funding for the Lingmuteychu Watershed Management Committee. In August 2006, the Small Grant Program of the Global Environmental Fund (SGP-GEF) of the United Nations Development Program (UNDP) formally accepted the project proposal and signed a memorandum of understanding with the watershed committee for the allocation of funding. These resources will be used for land and water conservation activities, in particular the restoration of an active landslide along the irrigation channel in Dompola village and tree plantings on degraded sloping land. 
Fig. 10. Facilitator declaring the collective impact after one time step.

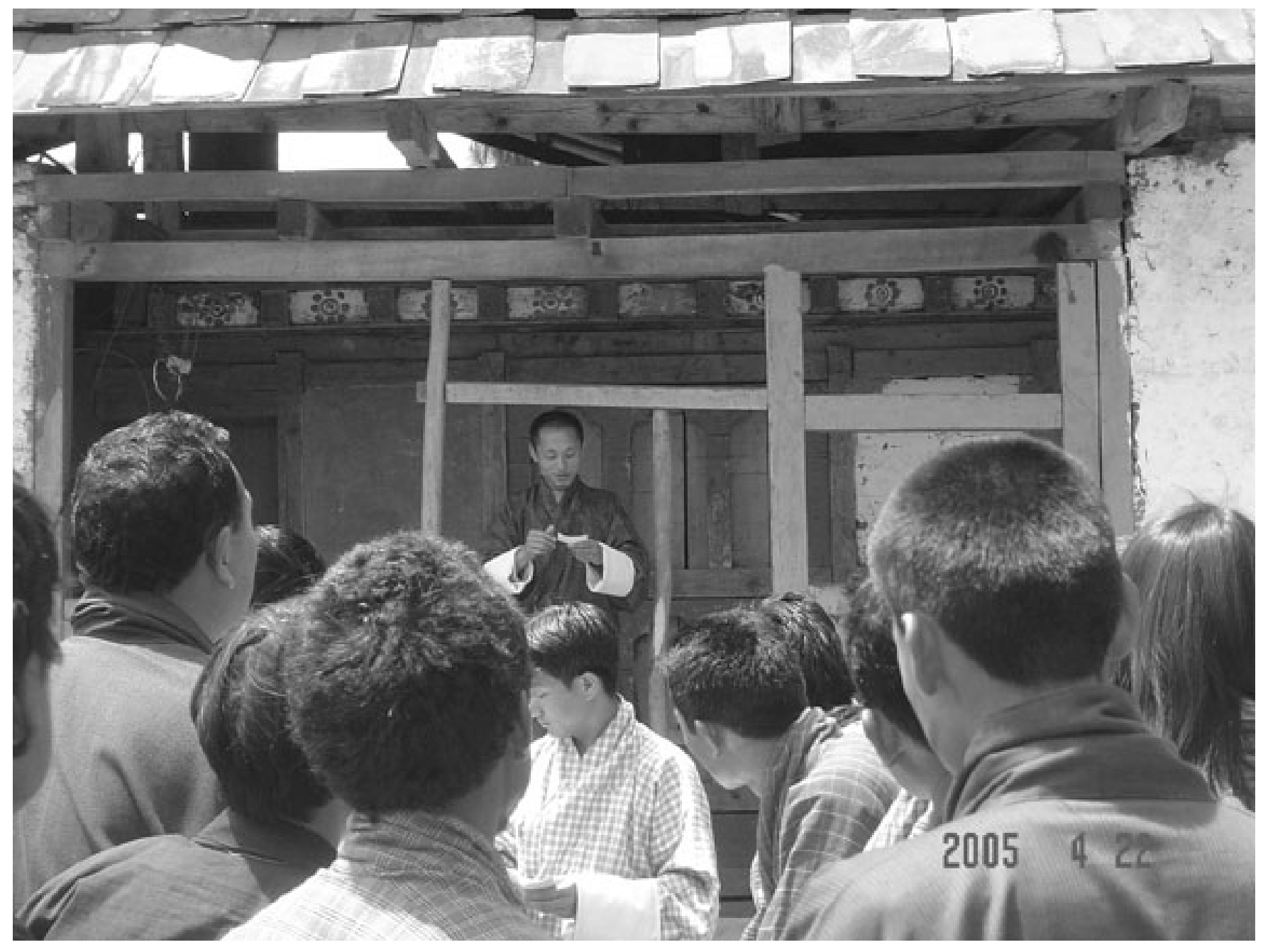

It is now planned to implement a simple model of the RPG in order to allow more people to play the game. The idea is to program a game similar to the RPG, with artificial agents using water; human players would be able to play with or against the artificial agents. Thus, the information and lessons can be disseminated to more people in the watershed. The model will be used to disseminate information rather than explore scientific results.
DISCUSSION: THE USE OF EMPIRICAL MODELS FOR CONFLICT RESOLUTION AND INSTITUTION BUILDING

In the ComMod process, we used two kinds of models, a computer simulation model and the RPG. The RPG may be considered as an open MAS model, in the sense that the environment is defined together with the agents, their roles, some of their actions and interactions, as well as the overall schedule of the agents' interventions. A degree of freedom is left to the players. 
Fig. 11. Water shared by the seven villages according to the various communication modes (average for four climates).

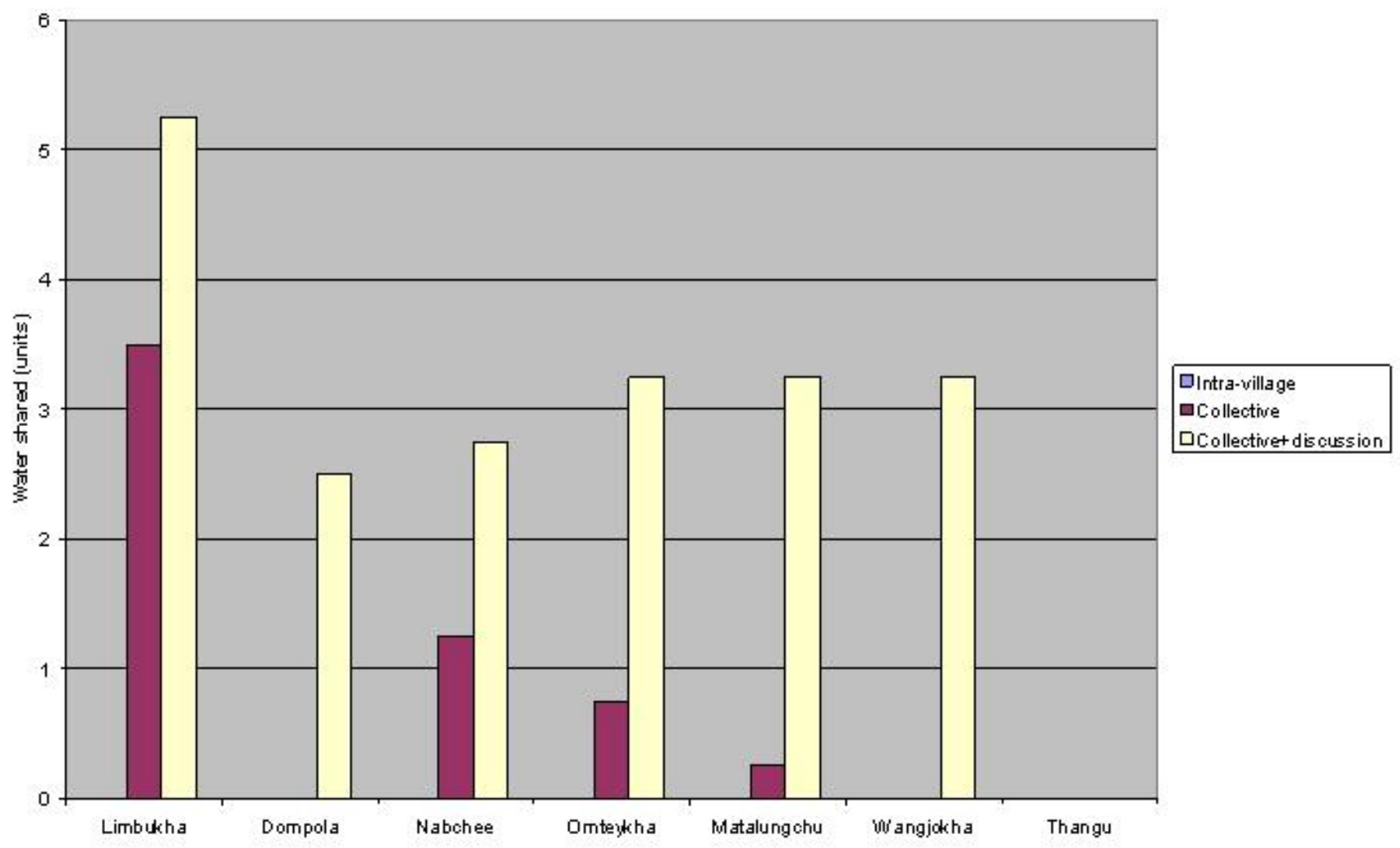

Three questions are addressed in this discussion. What is the connection between the model and reality, and how realistic should the model be? What are the roles and the uses of the model? What is the underlying social process that was accompanied by the researchers?

\section{Model vs. Reality}

The RPG was conceived from observation of reallife situations. In the first as well as the second RPG, an artificial environment is created (plots, crops, rainfall, market, etc.), the types of players are identified (the social context is taken into account, as well as the geographical setting), and the schedule of the different events is identified. One major point is that the model should be "playable." This means, for instance, that it is not possible to consider many time steps, or to have dozens of players. Thus, starting with the RPG implies that the model will be simple, and will not include many detailed processes. On the other hand, starting with the RPG provides an immediate test of the realism of the model, of an individual's behavior, and of the emergent process. When the players play the game, 
Fig. 12. Average individual income.

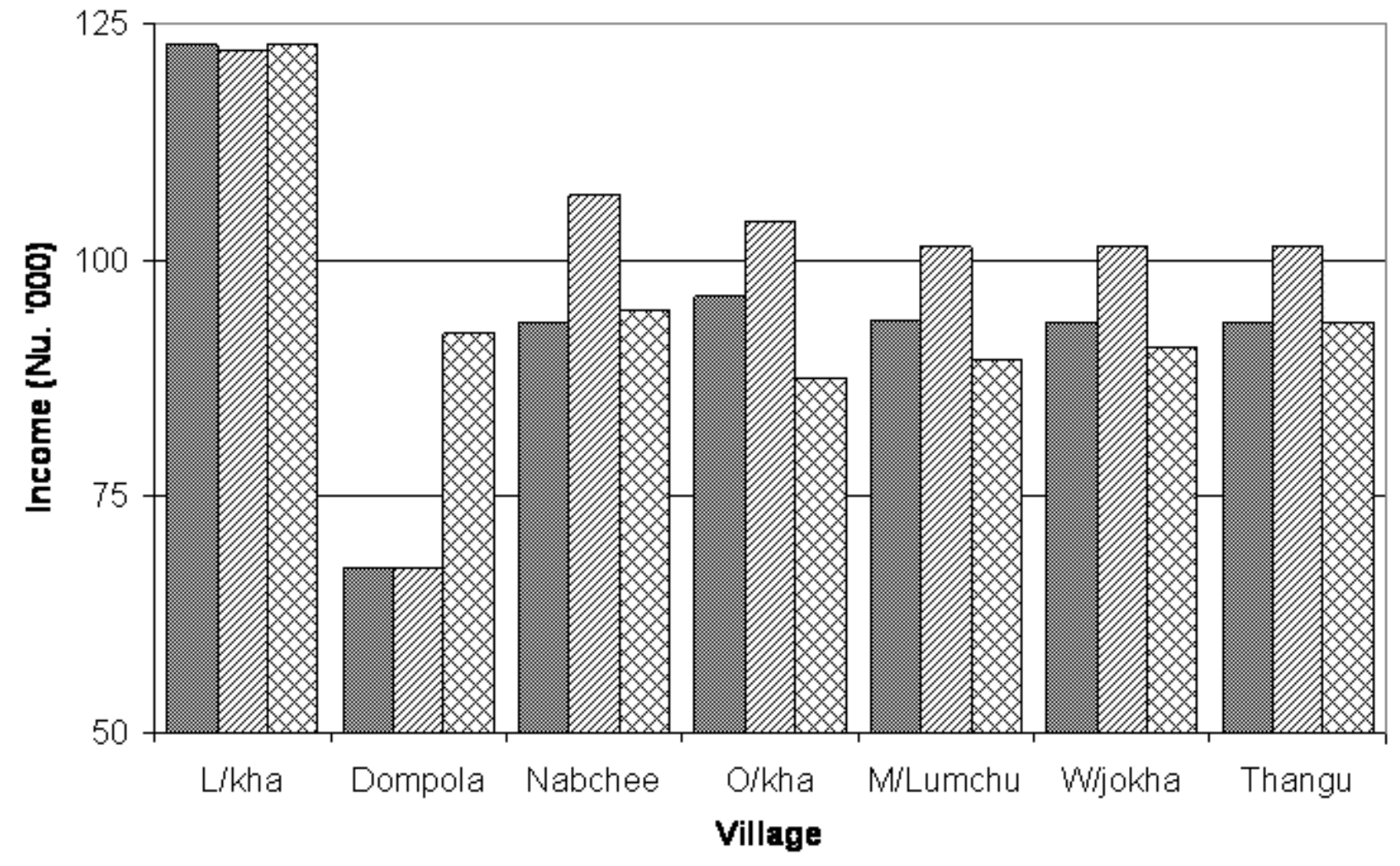

Individual $\square$ Collective $\nabla$ Swapped-Collective

they are able to comment on the actions that were planned for them. For instance, during the first RPG, players indicated that the RPG was not well conceived with regard to potato cropping. Players validate the behavioral rules, but more generally they also validate the model. They observe and comment the properties of the system emerging from the interactions among players (number of plots without water, number of water exchanges, etc.), and they can comment the links between these two organizational levels. Collective and individual interviews allow a better understanding of the decision-making process during the game. It is assumed that the decision-making process in the game is the same as that in reality. This has been assessed in many games.

Transcription of the model from the RPG implementation to computer simulation is often very easy. The most difficult part is to implement the decision-making process of the players. Again, the simpler the game, the easier the implementation of the decision-making process. When using MAS, the objective is not to implement a detailed decisionmaking process involving a lot of data and complex calculation, but rather to see how simple behaviors lead to complex phenomena. The example given in this paper shows how simple decisions, combined 
Fig. 13. Pledge to collectively manage the water.

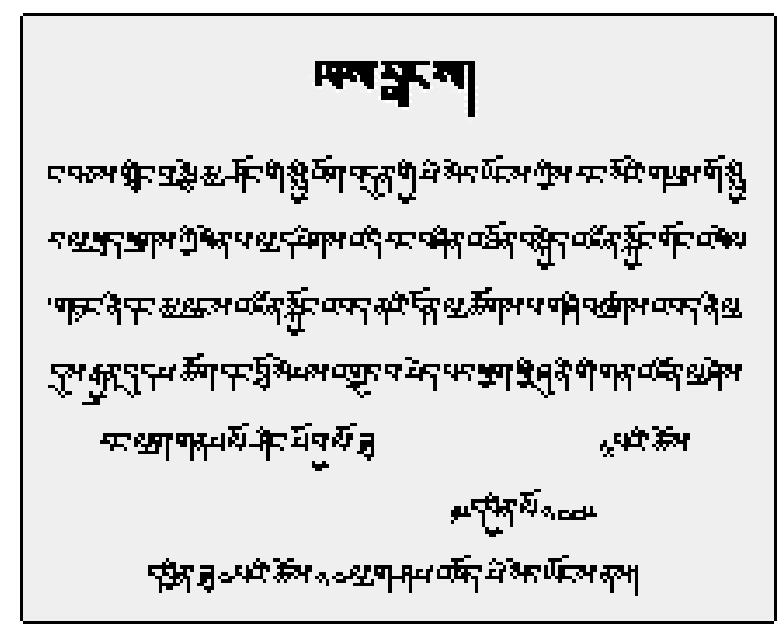

with different interaction protocols and different networks, lead to complex patterns that are meaningful for the stakeholders. In this case, the definition and calibration of the model, in brief its relationship with reality, was done with the objective of facilitating negotiation, which does not necessarily impliy a high realism. From our empirical studies, we came to the conclusion that very simple models with a low degree of realism can be very efficient.

\section{Use of the Model in the Negotiation Process: a Mediator Tool}

The example of Bhutan is one of the most advanced examples of the use of ComMod in a negotiation process. When the first workshop was organized, organizers were told that players would not even come because the conflict between Limbukha and Dompola was too strong. For many years, researchers had attempted to promote discussions among farmers without success. Two models were developed during this process in Bhutan, and these models were used as mediator tools.

The first model was used to facilitate discussions on water sharing between the two villages of Limbukha and Dompola. For many years, the root

\section{Pledge}

We the peqole of se ven tillages within the Lingmute ychu watershed, collectilely agree with byalty and dedication to manage the natial resoures and watershed for the present and the futire berests.

The agreement is signed on 15th day of 30' Lunar month of Female nood rooster year. the $24 h$ Apirl 2005. of the problem between these two villages has been the date at which Limbukha releases the water to Dompola. During the game, the two villages started to exchange water against money or labor. The discussions during the workshop after the game focused on the possibility of these exchanges in reality. However, some weeks after the game, the two villages started to discuss the date of water release. Again, they did not reach an agreement on the release date because the upper village claimed that no agreement had been formally established during the workshop. Thus, this was not a solution that emerged from the workshop and the use of the model remained in their mind rather than transferring to the negotiation process. This is a first indication that the model and the workshop are taken for what they are: a process and tools for facilitation among stakeholders but not an expert resource on technical solutions for a given problem. Similarly, the computerized model was used by the researchers to explore the various scenarios, comparing the effects of climate variability, the structure of social networks, and the communication protocols. The results show that the communication protocols are the most sensitive factor, leading to the conclusion that ca ommunication platform should be institutionalized to define collectively the best ways to share the water. 
Table 10. Course of action for the next 7 months

\begin{tabular}{|c|c|c|c|c|}
\hline \multirow[t]{2}{*}{ Activities } & \multirow[t]{2}{*}{ How to implement them } & \multirow{2}{*}{$\begin{array}{l}\text { Who will } \\
\text { implement them }\end{array}$} & \multicolumn{2}{|l|}{ Time frame } \\
\hline & & & Start & End \\
\hline $\begin{array}{l}\text { Appoint village representative to } \\
\text { drafting committee }\end{array}$ & Concerned villages & Village Tshokpa & $23 / 4 / 05$ & $30 / 4 / 05$ \\
\hline $\begin{array}{l}\text { Submit list of names for drafting } \\
\text { committee }\end{array}$ & Village Tshokpa & $\begin{array}{l}\text { Limbu Gup and } \\
\text { other two } \\
\text { villages' Tshokpa }\end{array}$ & & $30 / 4 / 05$ \\
\hline $\begin{array}{l}1^{\text {st }} \text { meeting to institute the } \\
\text { Lingmuteychu Watershed } \\
\text { management Committee }\end{array}$ & $\begin{array}{l}\text { Village committee } \\
\text { representatives }\end{array}$ & RNRRC Bajo & $13 / 5 / 05$ & $13 / 5 / 05$ \\
\hline $2^{\text {nd }}$ Meeting & $\begin{array}{l}\text { Village committee } \\
\text { representatives }\end{array}$ & RNRRC Bajo & $21 / 7 / 05$ & $31 / 7 / 05$ \\
\hline $\begin{array}{l}\text { Select and nominate Chairman and } \\
\text { members }\end{array}$ & Village Tshokpas & & Within 31/7/05 & \\
\hline $\begin{array}{l}\text { Awareness about outputs of the } \\
\text { workshop }\end{array}$ & All members & RNRRC Bajo & $23 / 4 / 05$ & Within 1 month \\
\hline Present final draft of the by-laws & Plenary (or workshop) & Drafting Committee & November 05 & \\
\hline Monitoring and Evaluation & Collective & RNRRC Bajo & & \\
\hline
\end{tabular}

Similarly, the second model was extremely simple. The seven villages have the same number of plots, there are almost no biophysical dynamics, and decisions to be made are very simple. It was used simply to introduce the idea of collective watershed management. The three scenarios led to the idea that a collectively managed watershed would iincrease benefits for the seven villages. Then, very detailed discussions were held among the stakeholders (farmers, governmental organizations) to reach an agreement on the establishment of a watershed committee. The first steps taken by the committee do not at all concern the sharing of water among villages, which was the topic of the game. Again the game and the model are taken for what they are: mediation tools. It shows that the stakeholders are in control of the negotiation process, and are able to see the difference between the model and reality. Thus, the risk of manipulation, which is a potential danger of this kind of method, is low.

\section{The Social Process}

In 1997, a first diagnostic study was done in the Lingmutyechu watershed, then research was started and the watershed became a pilot site for research in Bhutan. A lot of knowledge was accumulated about the ecological and agricultural systems, but conflict over water sharing was still reported. This is why, in 2003, researchers decided to conduct a companion modeling experiment. When the first RPG was decided upon, some people predicted that the villagers from the two conflicting villages would not even come. The farmers came. At start of the game, they immediately requested some changes, which were done. Then the farmers played, and even exchanged water among the two villages. The scenario with swapped roles, during which upper villagers played the role of the lower villagers and vice versa, was very effective at sharing different points of view. When a second RPG was organized in December 2003, participation was excellent, and 
from this, the farmers themselves asked for a workshop at the watershed level. During the watershed level workshop, people took the process very seriously: the farmers attending the workshop reported every evening to their villages. All officials from the districts, the villages, and the governmental organizations were present and participated. The workshop report was prepared by a group of participants and presented on the last day, and participants signed a document that planned the creation of a committee. Six months later, the committee actually created the watershed management committee. Throughout the process, researchers kept in touch with the farmers. After the first RPG started, specific monitoring was conducted. In addition, a student from the Communication and Innovation Studies lab of Wageningen University is conducting research on the evaluation of the process. Formal results are not available yet, but preliminary results provided to the RNRRC detail the main learning points by stakeholder category:

For the participants:

- The game setting promoted dynamic discussions among community members.

- It was only after the game session that the real implications of the game were realized; thus, the spontaneous actions and reactions generated new ideas that otherwise would not have emerged.

- The plenary sessions, gave a much-needed formal setting for concrete discussions on the outcomes of the RPG and for decision making.

- Players could use the RPG as means of communicating with their counterparts.

- The RPG seems, at first, like child's play, but it becomes a very strong tool to study complex interactions and dynamics.

For the facilitator:

- Alternating game sessions with plenary sessions allows people to relate the game to their real-life situation.

- Some local experience and knowledge is necessary in order to be able to facilitate the process.

- There is a definite need for skill to observe the behaviors and facilitate the process.

- The RPG can be used as a platform for conflict resolution.

- Mismanagement of the game can lead to a chaotic situation.

- The RPG effectively provides equal opportunities for all strata of players to participate in the game.

- It helps ordinary people put the NRM issues or problem in the right context for developing an appropriate strategy to address the problem.

\section{CONCLUSION}

This paper presents the use of multi-agent systems as tools to facilitate negotiation, in accordance with the companion modeling process. In the application presented, the conceptual model is first implemented as a role-playing game. The stakeholders play the game, thus validating the environment proposed, the behavioral rules, and the emergent properties of the game. It is then relatively easy to translate the RPG into a computerized MAS, which allows different scenarios to be explored.

Apart from this methodological aspect, this paper also present the use of such tools within the companion modeling methodology. The Bhutanese case study is a perfect example of the use of such tools for mediation purposes. It shows how the methodology helped transform a situation where there was conflict over sharing of water resources into a concrete agreement, culminating in the creation of an institution for collective watershed management.

Responses to this article can be read online at: http://www.ecologyandsociety.org/voll1/iss2/art36/responses/ 


\section{Acknowledgments:}

This research was supported by the International Rice Research Institute and CIRAD, and received financial assistance from the European Union, Asia It\&C project TH/Asia It\&C II/05 (96511). The contents of this document are the sole responsibility of the authors and can under no circumstances be regarded as reflecting the position of the European Union. The authors would like to thank the three anonymous reviewers, as well as Dr. B. Ekasingh, Dr. M Ekasingh, Aita Kumar Bhujel, Gyenbo Dorji, and Thinlay Gyamtscho for their help during this research.

\section{LITERATURE CITED}

Abel, N. 1999. Resilient rangeland regions. Pages 21-30 in D. Eldridge and D. Freudenberger, editors. Proceedings of the VIIth International Rangeland Congress. Townsville, Australia.

Barnaud, C., P. Promburom, G. Trébuil, and F. Bousquet. 2006. An evolving simulation and gaming process to facilitate adaptive watershed management in mountain northern Thailand. Simulation and Gaming: in press.

Barreteau, O., and F. Bousquet. 2000. SHADOC: a multi-agent model to tackle viability of irrigated systems. Annals of Operations Research 94:139162.

Berkes, F. 1997. New and not-so new directions in the use of the commons: co-management. The Common Property Resource Digest. Quarterly Publication of the International Association for the Study of Common Property 42:5-7.

Bhutan Water Partnership. 2003. Water resources management in Bhutan: a country status report. Thimphu, Bhutan.

Borrini-Feyerabend, G., M. Farvar, J. C. Nguiringuiri, and V. A. Ndangang. 2000. Comanagement of natural resources: organising, negotiating and learning by doing. IUCN, Gland, Switzerland; Cambridge, UK.
Bousquet, F., O. Barreteau, P. d'Aquino, M. Étienne, S. Boisseau, S. Aubert, C. Le Page, D. Babin, and J.-C. Castella. 2002. Multi-agent systems and role games: collective learning processes for ecosystem management. Pages 248285 in M. Janssen, editor. Complexity and ecosystem management: the theory and practice of multi-agent approaches. Edward Elgar Publishing, Cheltenham, UK.

Bousquet, F., O. Barreteau, C. Le Page, C. Mullon, and J. Weber. 1999. An environmental modelling approach. The use of multi-agents simulations. Pages 113-122 in F. Blasco and A. Weill, eds. Advances in environmental and ecological modelling. Elsevier, Paris, France.

Bousquet, F., O. Barreteau, C. Mullon, and J. Weber. 1996. Modélisation d'accompagnement : systèmes multi-agents et gestion des ressources renouvelables. In Quel environnement au XXIème siècle? Environnement, maîtrise du long terme et démocratie. (Proceedings, 8-11 September 1996, Abbaye de Fontevraud, France.)

Burton, M. 1994. The irrigation management game: a role playing exercise for training in irrigation management. Irrigation and Drainage Systems 7:305-348.

Castella, J. C., Tran Ngoc Trung, and S. Boissau. 2005. Participatory simulation of land-use changes in the northern mountains of Vietnam: the combined use of an agent-based model, a role-playing game, and a geographic information system. Ecology and Society 10(1):27. [online] URL: http://www.ecolog yandsociety.org/vol10/iss1/art27/.

Costanza, R., and M. Ruth. 1998. Using dynamic modeling to scope environmental problems and build consensus. Environmental Management 22 (2):183-195.

d'Aquino, P., C. Le Page, F. Bousquet, and A. Bah. 2003. Using self-designed role-playing games and a multi-agent system to empower a local decision-making process for land use management: the SelfCormas experiment in Senegal. Journal of Artificial Societies and Social Simulation 6(3):5. (online) URL: http://jasss.soc.surrey.ac.uk/6/3/5.html

Étienne, M. 2003. SYLVOPAST: a multiple target role-playing game to assess negotiation processes 
in sylvopastoral management planning. Journal of Artificial Societies and Social Simulation 6(2):5. (online) URL: http://jasss.soc.surrey.ac.uk/6/2/5.html

Étienne, M., C. Le Page, and M. Cohen. 2003. A step-by-step approach to building land management scenarios based on multiple viewpoints on multiagent system simulations. Journal of Artificial Societies and Social Simulation 6(2):2. (online) URL: http://jasss.soc.surrey.ac.uk/6/2/2.html.

Gonzalez, R. 2000. Platforms and terraces: bridging participation and GIS in joint learning for watershed management with the Ifugaos of the Philippines. Dissertation, ITC-Wageningen University, Wageningen, the Netherlands.

Gurung, T. R., and F. Turkelboom. 2000. A framework for community-based natural resource management for Bhutan. Discussion paper. Department of Research and Development Services, Ministry of Agriculture, Renewable Natural Resources Research Center, Khangma, Bhutan.

Holling, C. S. 1978. Adaptive environmental assessment and management. Wiley, London, UK.

Litmus Consult. 2002. Traditional water rights study. Integrated water resources management project. Ministry of Agriculture, Thimphu, Bhutan.

Lynam, T., F. Bousquet, P. d'Aquino, $O$. Barreteau, C. Le Page, F. Chinembiri, and B. Mombeshora. 2002. Adapting science to adaptive managers: spidergrams, belief models, and multiagent systems modeling. Conservation Ecology 5 (2):24 (online) URL: http://www.consecol.org/vol5/ iss $2 / \operatorname{art} 24$.

Manson, S. M. 2002. Validation and verification of multi-agent systems. Pages 63-74 in M. A. Janssen, editor. Complexity and ecosystem management. Edward Elgar Publishing, Cheltenham, UK.

McConnell, D. J., and J. L. Dillon. 1997. Farm management for Asia: a systems approach. FAO Farm Systems Management Series 13. FAO, Rome, Italy.

Meadows, D., and D. Meadows 1993. Fish Banks News. Fish Banks Ltd. and Laboratory for
Interactive Learning, University of New Hampshire, Durham, New Hampshire, USA,

Mermet, L. 1993. La nature comme jeu de société. L'Harmattan, Paris, France.

Messerschmidt, D., K. J. Temphel, J. Davidson, and I. W. D. 2001. Bamboo in the high forest of eastern Bhutan. International Center for Integrated Mountain Development, Kathmandu, Nepal.

Ministry of Agriculture. 2002a. Community-based natural resource management in Bhutan. Department of Research and Development Services, Ministry of Agriculture, Royal Government of Bhutan, Thimphu, Bhutan.

Ministry of Agriculture. 2002b. RNR Census 2000. Ministry of Agriculture, Royal Government of Bhutan, Thimphu, Bhutan.

National Environment Commission (NEC). 1998. The middle path: national environment strategy for Bhutan. NEC, Thimphu, Bhutan.

Piveteau, V. 1994. L'avenir à long terme des zones rurales fragiles, approche par le jeu prospectifd'une question complexe. Dissertation, Université de Paris, Paris, France.

Renewable Natural Resources Research Center. 1997. Characteristics of Lingmuteychu, problem diagnosis and major research themes. In Community-based natural resources management (CBNRM) research in Lingmuteychu watershed. RNRRC, Bajo, Bhutan.

Renewable Natural Resources Research Center. 1998. Program Annual report 1998. Ministry of Agriculture, Bajo, Wangduephodrang, Bhutan.

Röling, N. 1996. Towards an interactive agricultural science. European Journal of Agricultural Education and Extension 2(4):35-48.

Röling, N. 1999. Modelling the soft side of the land: the potential of multi-agent systems. Pages 73-97 in $\mathrm{C}$. Leeuwis, editor. Integral design: innovation in agriculture and resource management. Mansholt Institute, Wageningen, the Netherlands.

Royal Government of Bhutan. 2003. Bhutan 2003: people at the centre of development. Ministry of Finance, Thimphu, Bhutan. 
Trébuil, G., S. P. Kam, F. Turkelboom, and B. Shinawatra. 1997. Systems diagnoses at field, farm and watershed levels in diversifying upland agroecosystems: towards comprehensive solutions to farmers' problems. Pages 99-114 in P. S. Teng et al., editors. Systems approaches for sustainable agricultural development: applications of systems approaches at the farm and regional levels. Kluwer Academic Publishers and IRRI, Great Britain.

Tshering, D. 2001. Case study on bamboo and cane in Punakha and Wangdue valley. Renewable Natural Resources Research Center, Bajo, Bhutan.

Walters C. J., and R. Hilborn. 1978. Ecological optimization and adaptive management. Annual Review of Ecology and Systematics 9:157-188.

${ }^{[1]}$ A movie is available in Append. 4.

${ }^{[2]}$ Figs. 5, and A2.1-A2.8 are presented in Unified Modeling Language format, which is a standard for object-oriented design.

[3] Limbukha, Dompola, Nabchee, Omteykha, Matalunchu, Wangjokha, and Thangu.

${ }^{\text {[4] }} 1^{\text {st }}$ date $=\mathrm{n}^{\text {th }}$ day of the $4^{\text {th }}$ lunar month; $2^{\text {nd }}$ date $=10^{\text {th }}$ day of the $5^{\text {th }}$ lunar month; $3^{\text {rd }}$ date $=20^{\text {th }}$ day of the $5^{\text {th }}$ lunar month. (Last date for planting rice in the valley being $25^{\text {th }}$ July, RNRRC, Bajo.) 


\section{APPENDIX 1. THE LIMBUKHA-DOMPOLA RPG}

\section{The game board}

Two simple game boards (one for Limbukha and the other for Dompola) were drawn on a $0.5 \mathrm{~m} * 1 \mathrm{~m}$ poster paper representing the farmers in columns and their plots in rows (Fig. 3). On the game board, columns represented six farmers. For Limbukha, each column was divided into two subcolumns to represent potato (grown from March to June) and rice (grown from June to October). The game board for Dompola displayed just one column, implying that its farmers can grow only rice (June-October) and then fallow their fields (November-May).

Rows represent plots that ranged from 1 to 8 (depending on the category of the farmer). Each plot is equivalent to 0.1 ha of paddy field. Only one crop can be grown at a time. However, in the actual game, players proposed that Limbukha villagers could grow a crop of potato before any rice field. The year and cycle of the game (e.g., 4/2: implying year 4 and cycle 2 ) were indicated in the lower left corner of the board.

\section{The playing cards}

Six types of cards were used as a medium in the game:

- Name tag.

- Cash. Each player received initial cash to start farming at the following rates: Thruelpa $=\mathrm{Nu}$. $20,000(\mathrm{US} \$ 1=\mathrm{Nu} .47 .50)$, Chhep $=\mathrm{Nu} .15,000$, Chatho $=\mathrm{Nu} .10,000$, and Lhangchu $=\mathrm{Nu}$. 5,000 .

- Rainfall. Two cards normal (N) and low (L) rainfall for each cycle were used as chance cards to determine the volume of water available for sharing. Depending on the rainfall pattern, the units of water received by each player were regulated to induce dynamism. Before each cropping cycle, the card was randomly drawn and declared.

- $\quad$ Potato card. Limbukha farmers received yellow cards representing potato fields. One card was equivalent to 0.1 ha of potato grown before rice. Each player could use a maximum of three cards, and could also skip a season without growing potato.

- Water cards. Pink and light blue cards were used to represent water. One pink card was used as the equivalent to the volume of enough water to transplant and irrigate 0.1 ha of rice. Pink cards represented water used in the first cycle (first week of June to October) and light blue cards represented water used in the second cycle (third week of June to October). This means that farmers could place only one water card in one plot to indicate that that plot has been planted to rice. This card could be sold, exchanged, or used for transaction among villagers in a community or between farmers of the two communities. The game facilitator issued water cards in correspondence to the rainfall type. In the normal-rainfall season, Thruelpa received 5 water cards, Chhep 3 cards, Chatho 2 cards, and Lhangchu 1 card. During the low-rainfall pattern, the water provision was reduced by one unit, that is, 1 card less for each category.

- Market price. Two cards representing a high and low price were used to indicate potato and rice prices. One of these cards was drawn randomly and declared after each cycle. 


\section{The spreadsheet}

A spreadsheet program (Microsoft Excel) was used to record all the data produced from the RPG and to run simulations. The data from the game board were transferred into a data-capturing spreadsheet in codes $(1=$ rice, 2 = potato, and $3=$ fallow $)$. The data were linked to a simulation spreadsheet on which gross margin is analyzed. This spreadsheet was used to calculate income from land-use decisions. Based on the simulated results, each player was paid an income at the end of each game. Other data such as

water dynamics and land-use changes were analyzed after all the game sessions concluded. This actually facilitated the game session, thus enabling rapid calculations and inter-annual comparisons if required. 


\section{APPENDIX 2. THE COMPUTERIZED MODEL}

\section{The model structure}

\section{Spatial entities}

Spatial entities are made of elementary spatial entities and composite spatial entities. An elementary spatial entity represents the smallest homogenous unit of the environment in the model (a cell in CORMAS environment).

- Plot. In Limbukha model, the plot represents the elementary spatial entity. It is considered as the smallest homogenous unit that corresponds to the lowest land unit ( 1 langdo $=0.1$ ha) owned by any individual in Dompola and Limbukha. The basic interactions take place at plot level. The plot is characterized by 4 attributes: plot number, myblock (collection of plot belonging to one farmer), croppingpattern and crop. This entity undertakes only one operation (task) to update the status of the plot.

- Blocks. Each agent has a number of plots which are collectively represented as block. In Limbukha model there are 12 fields assigned to 12 farmers depending on their category. As the plots are components of block, the block is considered as composite spatial entity in Limbukha model.

\section{Passive objects}

In Limbukha model simple objects are rain, croppingPattern, crops, and market. The Message is also a passive object.

- Rain: the task of this object is to generate rainfall pattern for two cycles of the time step. There are two cycles in one time step, and each cycle can have either low or normal rainfall. It was done to relate the influences of rainfall on stream discharge and thereby irrigation water available.

- CroppingPattern: it is defined by either the potato-rice sequence OR the fallow-rice one depending on the rainfall pattern, market, and village conditions. It generates and initializes the crop succession for each time step.

- Crop: it is meant to define the crop type (potato or rice).

- Market: this object is meant to generate economic interactions. It is defined by 4 attributes (marketState, cropPriceKg, laborPriceHead, waterPriceUnit) and randomly generates market state as either low or high. It influences the economic calculation in the model and also the way players make their decisions regarding the crop succession for the next time step.

- Message: Any agent who needs to send message has to create an instance of a subclass of message and fulfill it. In Limbukha model there are 3 instances of message subclass and each subclass has a specific sequence of messages.

\section{Social agents}

- Farmer: in Limbukha model there are 12 farmers who communicate among agents and interact. Each agent is defined by attributes as given in Table A2.1. 
- Village: The 12 communicating agents are assigned to either of the villages. Farmer 1 to 6 represent Limbukha and 7 to 12 represent Dompola, which is similar to the RPG. The village is defined by one attribute name: either Limbukha or Dompola. The only task it has is to update water share among villager after rainfall is initiated.

Table A2.1. Attributes of a social agent (Farmer) in the Limbukha model

\begin{tabular}{|c|c|}
\hline Attributes & Explanation \\
\hline myWaterShareCategory & This attribute varies among communicating agents \\
\hline myField & Each agent has been assigned a field (from1 to 12) \\
\hline myLabor & $\begin{array}{l}\text { Represents number of units of labor an agent has. A thruelpa has } 60 \text {, cheep } 80 \text {, } \\
\text { chatro 180, and lhangchu } 160\end{array}$ \\
\hline myWater & $\begin{array}{l}\text { This is the unit of water share each agent has, depending on their category and } \\
\text { rainfall pattern for each cycle }\end{array}$ \\
\hline laborToBeExchanged & Excess labor that is available for exchange \\
\hline waterToBeExchanged & Unused irrigation water that is available for exchange \\
\hline laborExchanged & Number of work days received or given to AgentComm \\
\hline waterExchanged & Number of water shares received by or given to AgentComm \\
\hline myPotatoProduction & This represents the potato production class \\
\hline myRiceProduction & This represents the rice production class \\
\hline myMarket & This represents the market class (high and low) \\
\hline myIncome & This is the income gained in a year \\
\hline myVillage & This represents the village \\
\hline myCropSuccession & This represents the CropSuccession \\
\hline kinship & $\begin{array}{l}\text { This indicates who is related to whom, as kinship plays significant role in sharing } \\
\text { of irrigation water }\end{array}$ \\
\hline peopleToAskWater & A list of all farmers to ask for water \\
\hline twoCycleWaterExchanged & Sum of water exchanged in two cycles of a time step \\
\hline firstCycleWaterExchanged & Units of water exchanged in the first cycles of a time step \\
\hline acquaintancesLabor & Labor from acquaintances \\
\hline
\end{tabular}




\section{Model dynamics: the actions and interactions}

The behavior of agents can be classified into two broad categories: the agricultural methods and the communication methods.

Agricultural methods

In Limbukha model there are 8 tasks related to agricultural operations which an agent performs. Some of the major tasks of this model are explained below:

decideCroppingPattern: this is the first task that agent has to do. As depicted in Figure A2.1, agent makes decision on the crop succession that will be used in that time step.

calculateWaterLaborDemand: depending on the fallow land, crop succession, water and labor allowance, agent calculates the requirement of labor and water. This task will help to find quantity of labor and water available for exchange (Figure A2.2).

plantPotato: agents of only Limbukha plant potato in the first cycle of time step (Figure A2.3). plantRice: this task is used to plant rice in both villages in two cycles per time step (Figure A2.4).

exchangeWater: in this task agent who need water send messages and interactions take place among agents. If the agent does not get water the plot is left fallow.

harvestPotato: this task is undertaken at the end of the first cycle by Limbukha farmers only to remove (harvest) potato from the plots, such that it is free for planting rice in next the cycle. In the same task, yield of potato and income of farmer is updated.

harvestRice: this task is executed at the end of the second cycle of each time step when rice planted during both cycles are removed. During the same task, rice yield is updated followed by update of income. With this task the time step (or crop year) ends.

Fig. A2.1. Process for deciding on a cropping pattern in the Limbukha model. The dark dot represents the start of the process, the circled dot the end of the process.

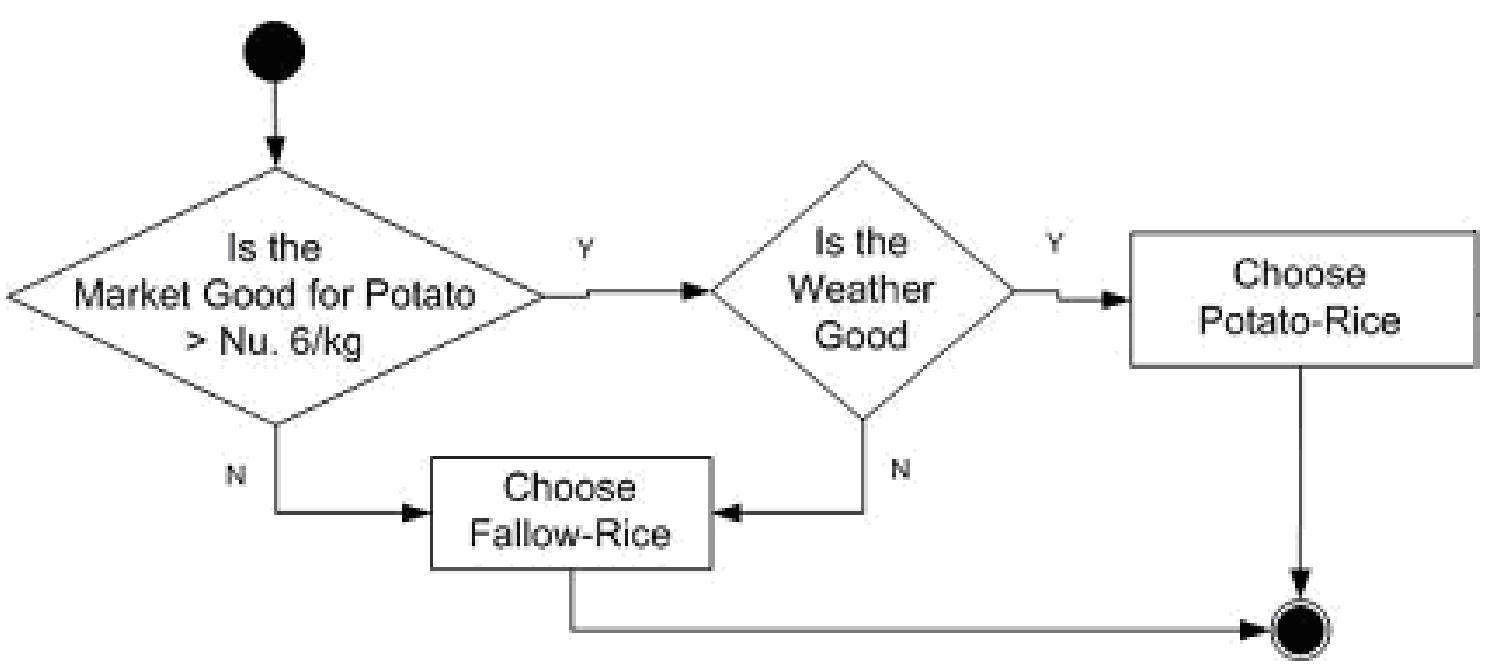


Fig. A2.2. Process for calculating demand for water and labor in the Limbukha model.

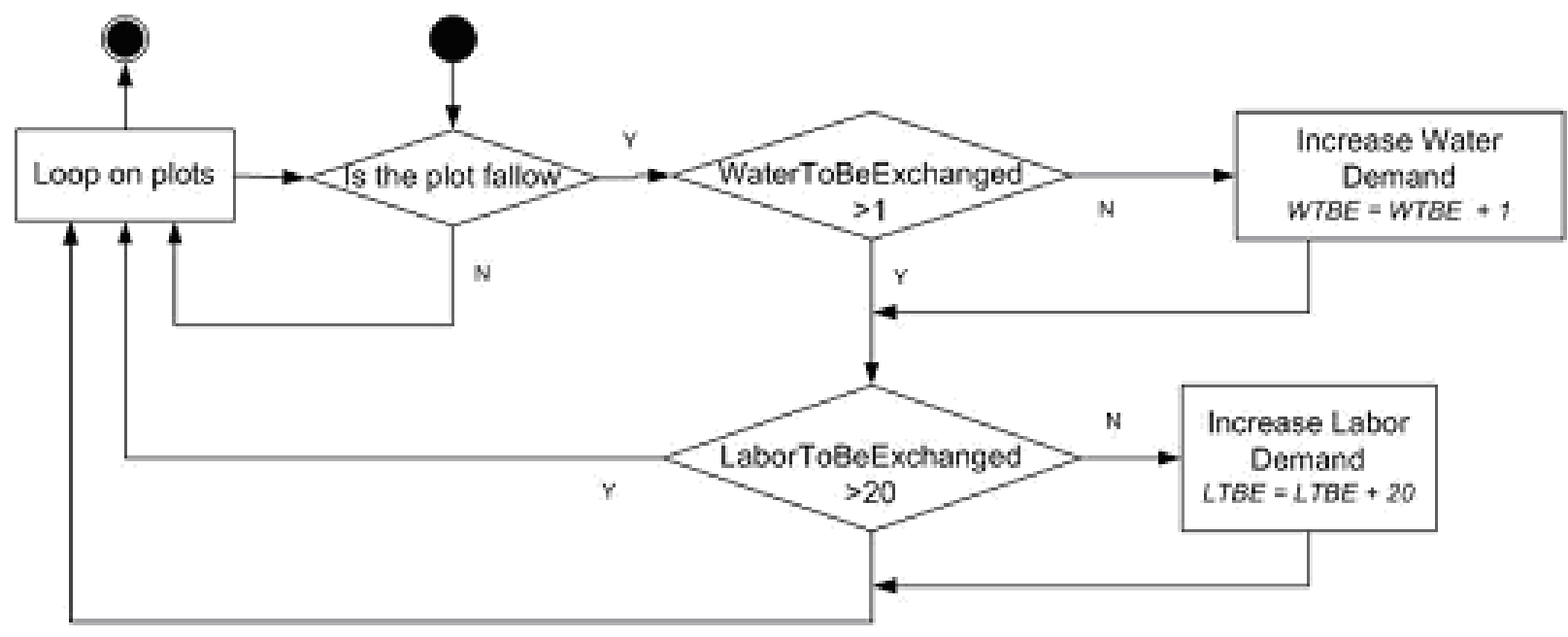

WTBE = Water to be exchanged $\mathrm{LTBE}=\mathrm{Labor}$ to be exchanged

Fig. A2.3. The "PlantPotato" task in the Limbukha model.

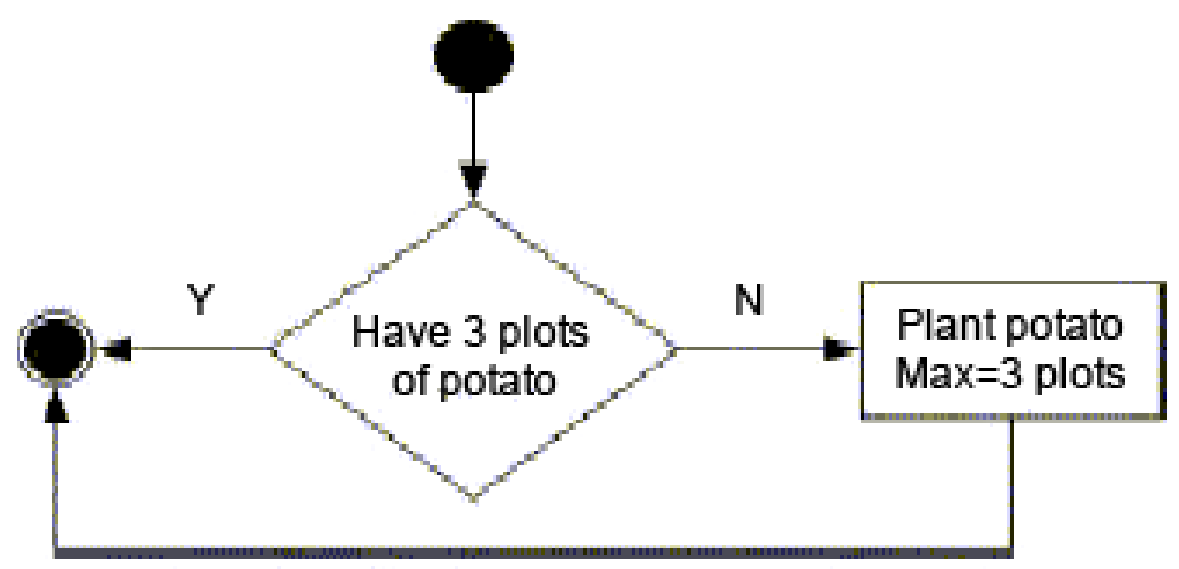


Fig. A2.4. The "PlantRice" task in the Limbukha model.

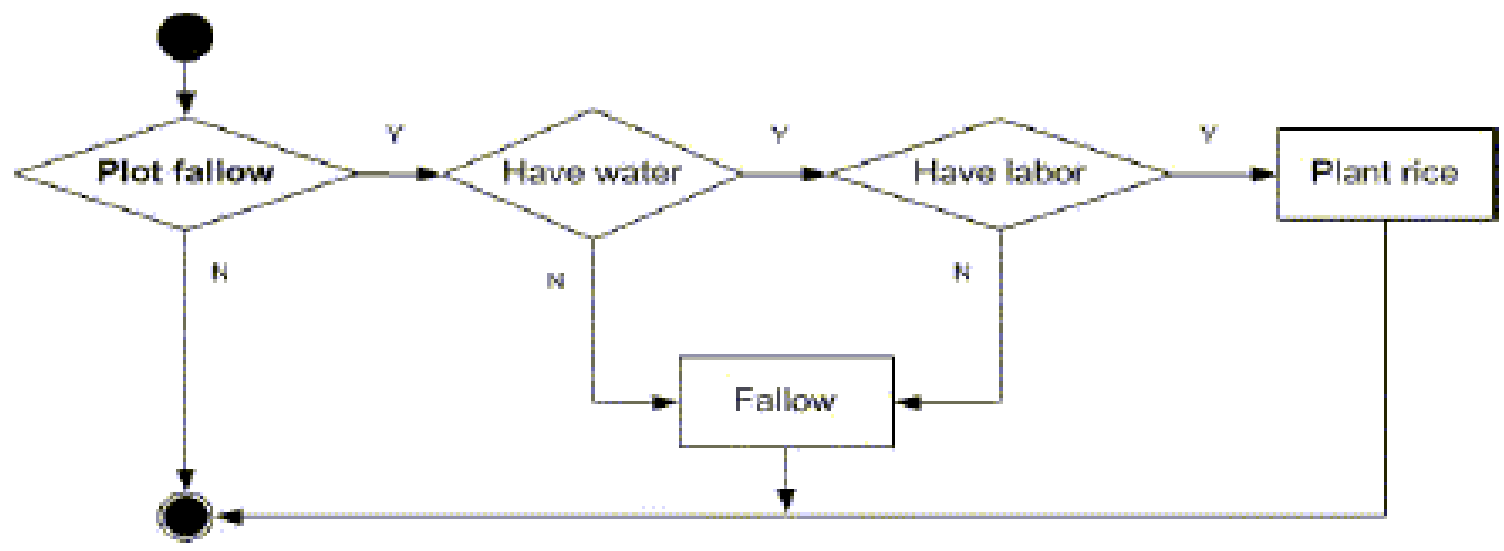

\section{Communication methods}

The dynamics of Limbukha model also depend on the way agents communicate among themselves to accomplish different tasks as explained in the preceding section. Firstly, the network of kinship within a village: where an agent identifies itself as kin to another agent and gives water free of cost whenever available. Secondly, agents communicate with acquaintances of their respective village. In the last method, they were allowed to communicate with agents of the other village. A protocol will be defined with three messages belonging to four kinds of actions.

1. Methods to define people to ask: the first step before any request for water or labor is requested, other agents of the network are defined either as kinship or acquaintance. From the acquaintance group, each agent defines the other members as those with whom they can interact for exchange of water and labor.

2. Methods to ask: in Limbukha model three messages have been programmed to ask water or labor. Messages like askLaborAcquaintances, askWaterAcquaintances, and askLaborAgainstWaterAcquaintances are associated to send in request for labor to acquaintances, water to acquaintances and asking labor against water respectively. All these messages are sent to the mailbox of all acquaintances asynchronously.

3. Methods to answer the request: in every time step, all agents check their mailbox for any message requesting water or labor. If the receiver has excess of labor or water, the agent sends a reply to the sender. In case there is no unused irrigation water or labor the receiver will not reply to the message.

4. Methods to supply: similar to replying to a message, the receiver sends in the requested number of labor or unit of water to the sender of the message. There are instances where receiver make return request for labor against water or even cash. The sender will pay back according to the request. Both receiver and the sender will update the account of labor, water and income. 
Agents may exchange either within a kinship network or among an acquaintance network. In this study 6 different protocols of interactions have been identified. The protocol that resembles reality to a certain extent is presented in Figure A2.5 which shows how agents " $A$ " interact with agent " $B$ " to get water.

Fig. A2.5. Protocol for exchange of water and labor in the Limbukha model.

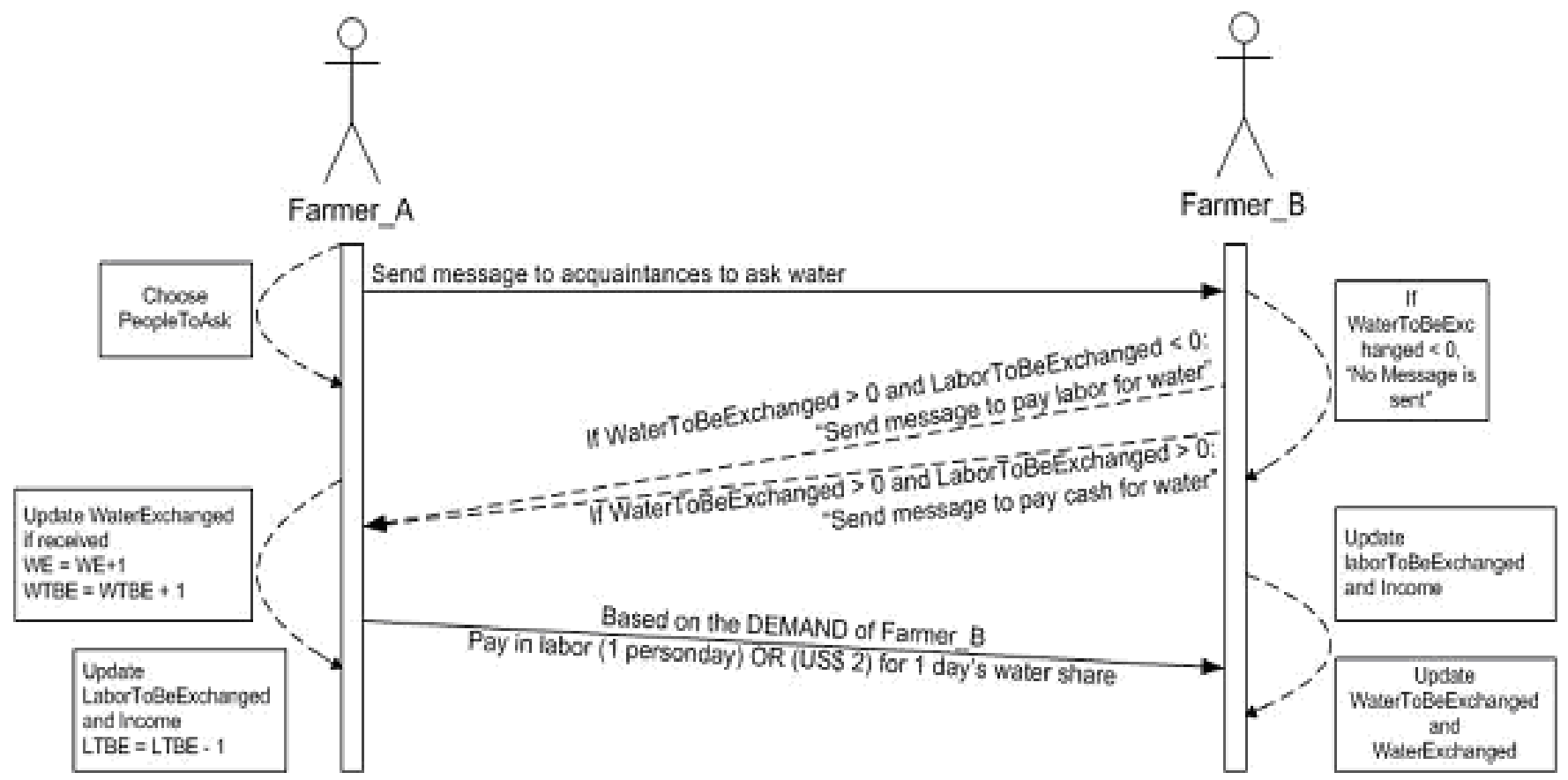

\section{Model dynamics: the scheduling}

The sequence diagram shows how objects communicate with one another over time. The key idea here is to show the interactions among objects taking place in a specific sequence. For building the Limbukha model, the base sequence was constructed using lessons learned from RPG (Figures A2.6 and A2.7). Here, one time step is equivalent to 1 year, each time step is divided into two cycles. 
Fig. A2.6. Sequence diagram of Limbukha model (Cycle1 corresponding to January to mid-June).

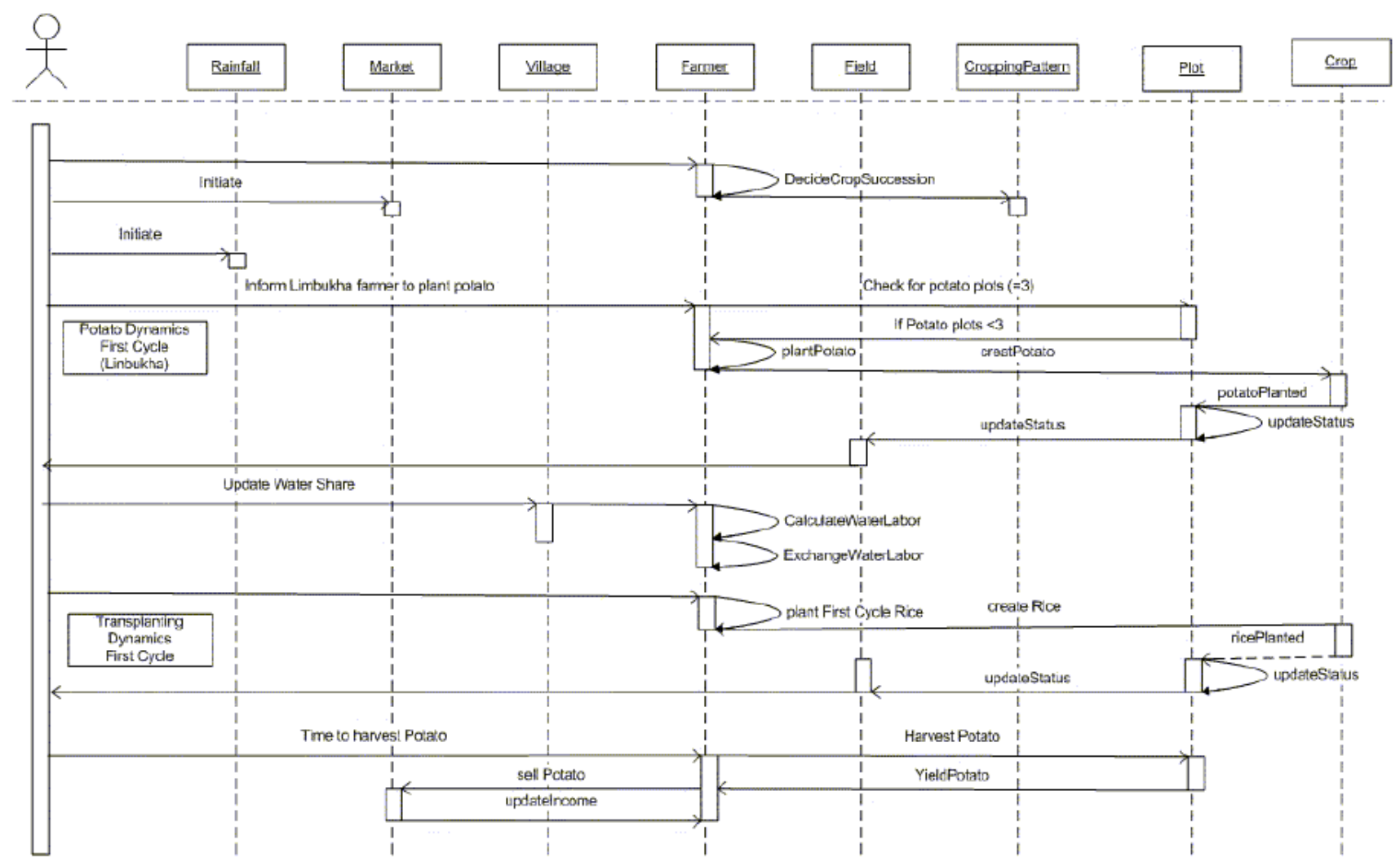

Fig. A2.7. Sequence diagram of the Limbukha model (Cycle 2 corresponds to mid-June to December).

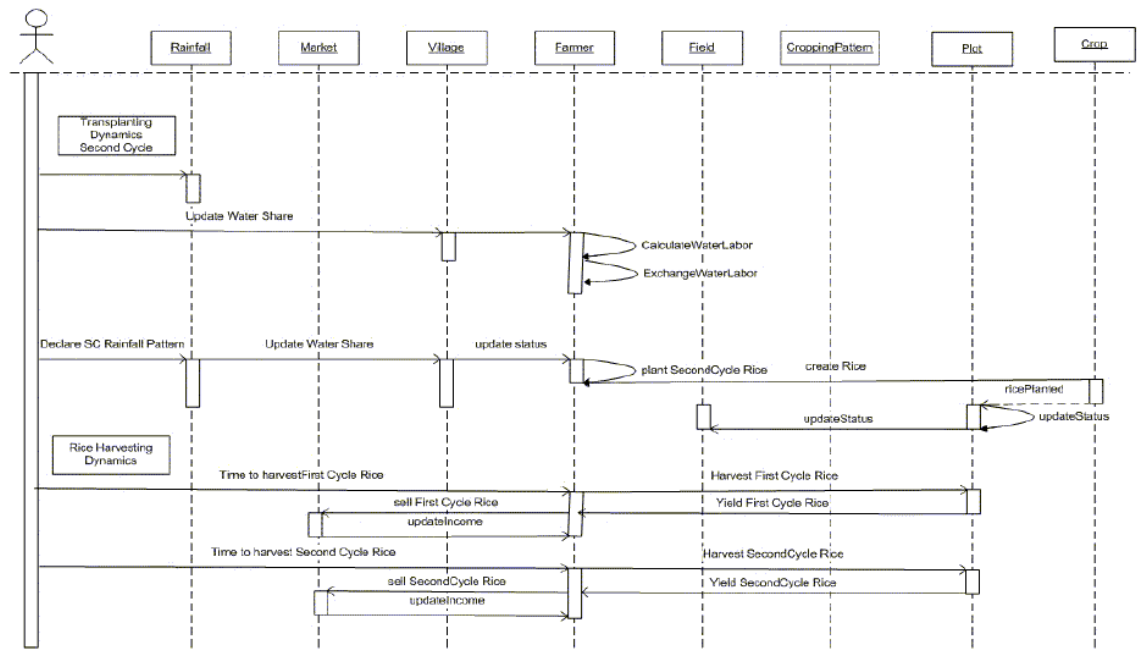


Cycle 1

All farmers decide on the crop succession based on the rainfall and market status.

Market price is updated to inform on the last year's market state.

Rainfall is initiated for the first cycle (January to mid June).

The information on rainfall pattern in given to the villages. At village level water is updated and allocated to each farmer based on his or her category and rainfall pattern. Each farmer calculates his water needs and exchanges with other farmers.

Limbukha Farmers only plant potato in their plots (maximum of 3 plots per farmer).

Farmers of both villages plant rice.

Limbukha Farmers whoever planted potato (in step 5) are activated to harvest (remove) potato and update their plots as fallow. In the same sequence they sell their potato harvest and update their incomes.

\section{Cycle 2}

Rainfall is initiated for the second cycle (mid June to December).

The information on rainfall pattern in given to villages. At village level water is updated and allocated to each farmer based on his or her category and rainfall pattern. Each farmer calculates his/her water needs and exchanges with other farmers.

Farmers from both villages are activated to plant rice.

Farmers from both villages harvest (remove) rice and update their plots/block as empty. In the same sequence they sell their harvest rice and update their income.

\section{Implementation with Cormas}

Programming was done in CORMAS[1] (Bousquet et al., 1998). The artificial environment was designed to represent plots and blocks of plots assigned to 12 farmers. For the synthetic environment an interface of $8 \times 13$ grid size was used (Figure A2.8). It was like placing two game boards (one for Limbukha and other for Dompola) used in Dompola RPG side by side. Field 1-6 represents Limbukha while 7-12 represents Dompola.

Two modes of communication (intra-village and inter-village, Scenarios N2 \&N3 in Table 2) were tested. In each time step it was seen an interface shows which agents are communicating. Figure A2.9 shows the exchange of water between farmer 7 and 9; 7 and 10 and 4 and 2 .

Fig. A2.8. The artificial "Synthetic" environment and main grid interface of the Limbukha model. Green shapes represent rice crops and dark orange shapes represent potato crops. 
Fig. A2.9. The CORMAS Communication observer showing the exchange of water between agents in Limbukha Model (circle represents communicating agents). Blue triangles represent the Farmer agents, red triangles represent the Village agents.

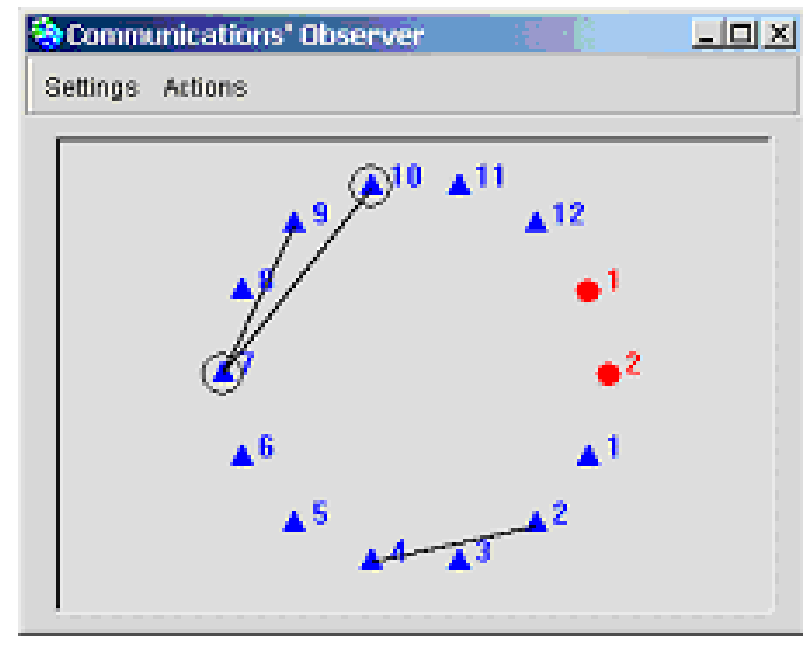

[1] http://cormas.cirad.fr 


\section{APPENDIX 3. THE SEVEN VILLAGES’ RPG AND WORKSHOP SETTINGS}

\section{Role playing game description}

Although the mode of communication and players varied across games, there were some common features in all games. The pertinent features common to all games are as follows:

\section{Game board}

In all the games, a wooden game board resembling the ones used for tradition game tiger and cattle " $t a$ da-no" was used (Figure A3.1). To suit for the game, the board had 12 hollow outs designed to represent 12 plots (or terraces). In one corner of the board, a rectangular depression (1"x2"x0.5") was made to represent irrigation channel where village level water share was placed during each time step. The other end of the board has two rectangular hollow out, one representing water received from other village and the other to place any balance water share. In all the games number of players were kept 3 such that it matched the basic design of board where only three could play. Each player was allocated 4 plots and each village had 12 plots of paddy. For the purpose of calculation, one plot was considered equivalent to 0.1 hectare of land.

\section{Water share}

Small wooden cubes $\left(1 \mathrm{~cm}^{3}\right)$ were used to represent water share in the game. One cube meant 1 unit of water enough to grow rice in one plot on the game board. Water share per village was predefined as shown in Table 8 which was dependent on weather types. It was left to the players to discuss and share the allocated water share within the village.

Fig. A3.1. Game board representing a village irrigation scheme.

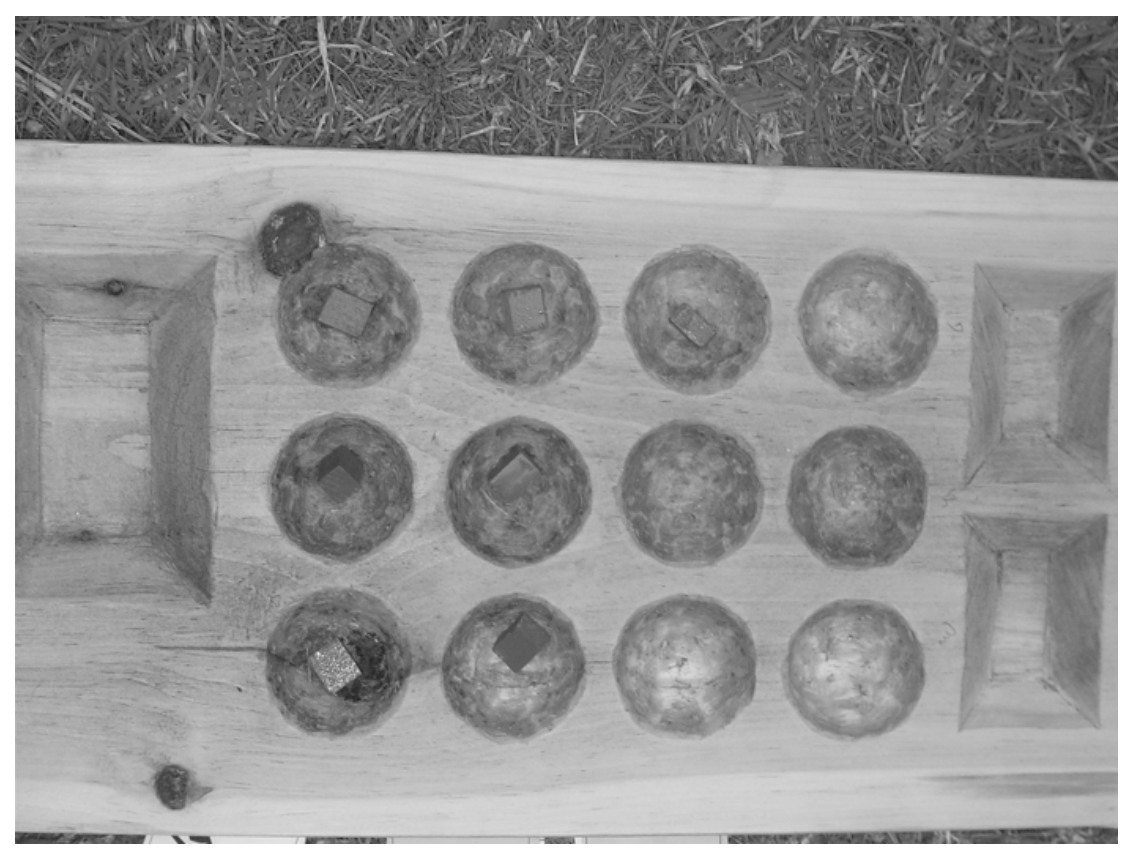




\section{Workshop settings}

\section{Participants}

Each village was represented by 3 members. These 3 players per village were Village representative (Tshogpa), Chusup (Village water guard), and a farmer (active irrigator). They were selected based on the following requirements:

- Person knowledgeable about the irrigation canal, water sharing systems and issues related to systems operations (Chusup),

- Person who is knowledgeable about the village in general, development needs and constraints, and has a decision making role in the village (Tshogpa), and

- Person who is an active farmer knowing about the issues related to irrigation water and rice cultivation (irrigator).

\section{Process}

A generic game representing the watershed was used where 3 players each from 7 communities participated in the game. To ensure the smooth course of the process, 7 group facilitators, 2 game facilitators, 4 observers and 1 overall coordinator was engaged during the game. The workshop lasted for 4 days, structured into first 2 days for RPG, day 3 for interview and discussion, and the preliminary results and workshop recommendations were presented to all the participants on $4^{\text {th }}$ day. The workshop process can be summarized as follows:

1. The workshop started with general briefing, explaining the objectives of the workshop. The process of the game was also explained in detail. To provide a technical explanation on watershed from hydrological perspectives, an overview of the hydrological cycle and the concept of watershed were presented.

2. Three modes of communication (Intra-village, collective with flow of excess irrigation water, and collective decision on water sharing) were played.

3. Players were assigned to their respective villages, and explained their roles as irrigators. One facilitator was attached to each group.

4. On day 1, five rounds of individual mode were played, where players were allowed to discuss and exchange water exclusively within their group.

5. In the afternoon of Day 1, 5 rounds of collective mode with flow of any surplus water were played.

6. The second collective mode was played on Day 2. During late afternoon of Day 2, the report drafting team (mangmi, clerk of Gup, some voluntary farmer representatives, District officers, Extension staff and researchers) brainstormed on the development of watershed level management committee,

7. On Day 3 the Tshogpa of 7 villages, gup, mangmi, dungyee, District officers, Extension staff and researchers were requested to report. Each community members present on Day 3 was interviewed on their views on the RPG and the process. Following the interview, the group deliberated on the Lingmutey Chu Watershed Management Committee. 
8. The preliminary result of the RPG and recommendations of the group discussion were presented to the plenary on Day 4. 


\section{APPENDIX 4. A MOVIE ON THE COLLECTIVE WORKSHOP TO FACILITATE DISCUSSION} BETWEEN TWO VILLAGES

Erratum: There is a mistake in the figure of the movie. The correct figure can be found in Fig. 4 in the paper.

Click here to download and play movie. Because of the large file size, download of this movie may take more than 2 minutes. (File type: AVI, File size: $36206 \mathrm{~KB}$ ) 